TITLE:

\title{
Bisection algorithm for computing the frequency response gain of sampled-data systems - Infinite- dimensional congruent transformation approach
}

$\operatorname{AUTHOR}(S)$ :

Ito, Y; Hagiwara, T; Maeda, H; Araki, M

\section{CITATION:}

Ito, $Y$... [et al]. Bisection algorithm for computing the frequency response gain of sampleddata systems - Infinite-dimensional congruent transformation approach. IEEE TRANSACTIONS ON AUTOMATIC CONTROL 2001, 46(3): 369-381

ISSUE DATE:

2001-03

URL:

http://hdl.handle.net/2433/39970

\section{RIGHT:}

(c)2001 IEEE. Personal use of this material is permitted. However, permission to reprint/republish this material for advertising or promotional purposes or for creating new collective works for resale or redistribution to servers or lists, or to reuse any copyrighted component of this work in other works must be obtained from the IEEE. 


\title{
Bisection Algorithm for Computing the Frequency Response Gain of Sampled-Data Systems- Infinite-Dimensional Congruent Transformation Approach
}

\author{
Yoshimichi Ito, Tomomichi Hagiwara, Member, IEEE, Hajime Maeda, Member, IEEE, and \\ Mituhiko Araki, Member, IEEE
}

\begin{abstract}
This paper derives a bisection algorithm for computing the frequency response gain of sampled-data systems with their intersample behavior taken into account. The properties of the infinite-dimensional congruent transformation (i.e., the Schur complement arguments and the Sylvester law of inertia) play a key role in the derivation. Specifically, it is highlighted that counting up the numbers of the negative eigenvalues of self-adjoint operators is quite important for the computation of the frequency response gain. This contrasts with the well-known arguments on the related issue of the sampled-data $H_{\infty}$ problem, where the key role is played by the positivity of operators and the loop-shifting technique. The effectiveness of the derived algorithm is demonstrated through a numerical example.
\end{abstract}

Index Terms-Bisection algorithm, frequency response gain, numerical computation, sampled-data systems, Schur complement arguments.

\section{INTRODUCTION}

$\mathbf{T}$ HE widespread use of digital controllers has stimulated modern treatment of sampled-data systems, in which we can take into account their intersample behavior directly and exactly (see, e.g., [6] for an overview). Among important landmarks in the recent development of modern sampled-data control theory is the study on the frequency response of sampled-data systems. What distinguishes this notion from the classical frequency response of discrete-time systems is that the new notion is defined between the continuous-time input $w$ and the continuous-time output $z$ of the internally stable sampled-data system as shown in Fig. 1, consisting of the continuous-time plant $P$, the discrete-time controller $\Psi$, the hold device $\mathcal{H}$ and the sampler $\mathcal{S}$ with sampling period $h$. Such a notion has been introduced with the lifting approach [32], and independently with the FR-operator approach [1], and it is known that the definitions in these two approaches are essentially equivalent [30].

Manuscript received February 11, 1999; revised November 30, 1999 and August 1, 2000. Recommended by Associate Editor T. Chen.

Y. Ito and H. Maeda are with the Department of Communications Engineering, Osaka University, Osaka, 565-0871, Japan (e-mail: ito@ comm.eng.osaka-u.ac.jp; h-maeda@comm.eng.osaka-u.ac.jp).

T. Hagiwara and M. Araki are with the Department of Electrical Engineering, Kyoto University, Kyoto, 606-8501, Japan (e-mail: hagiwara@kuee.kyoto-u.ac.jp; araki@kuee.kyoto-u.ac.jp).

Publisher Item Identifier S 0018-9286(01)02561-2.

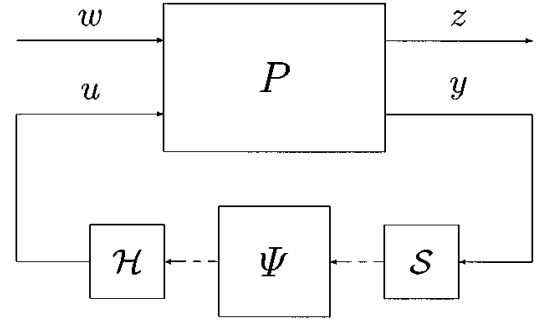

Fig. 1. Sampled-data control system.

The frequency response of sampled-data systems is an important notion as well as a useful tool, since it has close relationships, e.g., to the $H_{2}$ and $H_{\infty}$ problems of sampled-data systems [4]-[6], [11], [16], [18]-[20], [28], [29], [31], the robust stability problem against LTI perturbations [8], [12], [23], and the digital redesign of continuous-time controllers [25]. However, the computation of the frequency response gain of sampled-data systems corresponds to that of the norm of an infinite-dimensional operator, and is a nontrivial matter.

Regarding this tough problem, Yamamoto and Khargonekar [32] gave a quite neat closed-form solution based on a certain $\gamma$-dependent generalized eigenvalue problem. To compute the frequency response gain numerically based on this result, however, we need to carry out a one-dimensional search with respect to $\gamma$, and it involves a numerically undesirable process of zero/nonzero judgment (see Section IV-A). Also, it is generally time-consuming.

There are a few other methods used to compute the frequency response gain approximately [1], [15], [33], but they require to compute the norm of a huge matrix as we increase the degree of the approximation, which is undesirable from the viewpoint of computational load and numerical reliability. A different type of efficient approximation method to compute the frequency response gain was given recently in [14]. This method has advantages in the above viewpoint, and seems to give satisfactorily accurate results in many cases, but it also has a disadvantage that there is no simple way to improve accuracy.

In any case, however, it is easy to compute, with these approximate methods or another, a set of fairly good upper and lower bounds of the frequency response gain of sampled-data systems. Given this fact, a bisection algorithm for the computation of the frequency response gain of sampled-data systems is 
highly desirable from the viewpoint of computational load and numerical reliability. However, the previous attempts [13], [15] to derive such an algorithm have been successful only partially as explained later in this Introduction, and deriving a complete bisection algorithm still remains one of the most important open problems in the modern sampled-data control theory. Now, the purpose of this paper is to derive such an algorithm, and demonstrate its effectiveness through a numerical example.

Essentially, deriving a bisection algorithm boils down to deciding whether or not the frequency response gain at each frequency is smaller than a given positive number $\gamma$. In this paper, we exploit some useful properties of the infinite-dimensional congruent transformation (i.e., the Schur complement arguments and the Sylvester law of inertia) to reduce this binary decision into counting up the number of the negative eigenvalues of a certain block-diagonal self-adjoint operator consisting of two $\gamma$-dependent blocks: a finite-dimensional matrix block and an infinite-dimensional operator block. Here, the former can be computed with the finite-dimensional state-space matrices of the sampled-data system by an exponentiation formula, so that its negative eigenvalues are easy to count. On the other hand, counting up the number of the negative eigenvalues of the latter block (i.e., the infinite-dimensional operator block) amounts to counting up the number of the singular values of $\mathcal{D}$ larger than $\gamma$ where $\mathcal{D}$ denotes the direct feedthrough term in the lifted representation of the sampled-data system. The computation of these singular values could be carried out with an existing method [5], but this paper further elaborates on deriving a much more reliable method (actually, a bisection algorithm) for their computation, again through essentially the same technique with the infinite-dimensional congruent transformation. Thus, we can readily obtain a bisection algorithm for the computation of the frequency response gain.

As seen from the above, the arguments in this paper contrast with the well-known arguments on the related issue of the sampled-data $H_{\infty}$ problem [4], where the positivity of operators and the loop-shifting technique play a key role. Instead, in this paper, a key role is played by a more general idea of the numbers of the negative eigenvalues of operators, and the properties of the infinite-dimensional congruent transformation are the crucial tool for the developments. Because of this difference in the underlying tools, we do not need to make the assumption on $\gamma$ that $\gamma>\|\mathcal{D}\|$, which corresponds to the positivity condition of a certain operator and was made in the previous attempt toward a bisection algorithm [15] — since the frequency response gain can actually be smaller than $\|\mathcal{D}\|$, such an assumption is not appropriate and, hence, the previous attempt was only partially successful. The other attempt given in [13] was not completely successful, either, since it made a strong assumption on $P$, but the bisection algorithm derived in this paper can be regarded as a natural extension to it.

This paper is organized as follows. In Section II, we give the notation used in this paper and provide some useful fundamental results regarding the infinite-dimensional congruent transformation of operators. Section III constitutes the main part of the paper, where we first review the notion of the frequency response of sampled-data systems based on the lifting approach, and then we provide our main results on the bisec- tion algorithm for the computation of its gain. The state-space formulas for the computations involved in the algorithm are also given there. Next, in Section IV, we study some related issues. Namely, in Section IV-A, we discuss some important implications of our arguments in Section III, and clarify the relationship of the proposed bisection algorithm to one-dimensional search algorithms, especially the existing one derived by Yamamoto and Khargonekar [32]. Next, in Section IV-B, we study the relationship to the task of solving the sampled-data $H_{\infty}$ problem. The arguments there provide a clearer link between this task and the derivation of the bisection algorithm. In Section IV-C, we give some remarks about the parallel arguments with the FR-operator approach, and show that we can derive a different but equally useful bisection algorithm for the computation of the frequency response gain. In Section IV-D, we give some possible applications of our infinite-dimensional congruent transformation approach developed in this paper. Finally, in Section V, we study a numerical example, and demonstrate the effectiveness of the proposed bisection algorithms.

\section{PRELIMINARIES}

In this section, we give the notation used in this paper and prepare some useful results for computing the frequency response gain of sampled-data systems.

The space of square integrable functions over the time interval $[0, h)$ equipped with the usual inner product will be denoted by $L_{2}[0, h)$. The direct sum of $m$ such spaces, $\left(L_{2}[0, h)\right)^{m}$, is also denoted by $L_{2}[0, h)$ for notational simplicity. The $n$-dimensional complex Euclidean space will be denoted by $\mathbf{C}^{n}$. It is well-known that $L_{2}[0, h)$ and $\mathbf{C}^{n}$ as well as their direct sum are separable Hilbert spaces. Let $\mathcal{M}$ be a separable Hilbert space. For $x, y \in \mathcal{M}$, their inner product is denoted by $\langle x, y\rangle$, and the norm of $x \in \mathcal{M}$ is denoted by $\|x\|$. The adjoint of a linear operator $\mathbf{Q}$ on $\mathcal{M}$ is denoted by $\mathrm{Q}^{*}$, and the transpose of a matrix $P$ is denoted by $P^{T}$. The identity operator or matrix is denoted by $I$. The class of linear self-adjoint compact operators on $\mathcal{M}$ is denoted by $\mathcal{W}$. For $\mathbf{X} \in \mathcal{W}$, it is well-known [22] that i) every $\lambda$ in the spectrum is a real number, and is in fact an eigenvalue provided that $\lambda \neq 0$, ii) the eigenvalues are at most countably infinite, ${ }^{1}$ and iii) 0 is the only possible point of their accumulation. For a linear compact operator $\mathbf{X}$ on $\mathcal{M}, \sigma_{i}(\mathbf{X})$ denotes the $i$ th largest singular value of $\mathbf{X}$, which is given by the square root of the $i$ th largest eigenvalue of $\mathbf{X}^{*} \mathbf{X}$. The class of the operators of the form $\gamma I-\mathbf{X}$ ( $\mathbf{X} \in \mathcal{W}, \gamma$ is a positive number) is denoted by $\mathcal{N}$. Note that every linear self-adjoint operator on a finite-dimensional space $\mathcal{M}$ belongs to $\mathcal{N}$, even with a prescribed value of $\gamma$, and this fact will be used throughout the paper. If the underlying space $\mathcal{M}$ is infinite-dimensional, on the other hand, every $\mathbf{Y} \in \mathcal{N}$ can be expressed uniquely as $\mathbf{Y}=\gamma I-\mathbf{X}$, and thus is noncompact, but has the property that i)' every $\lambda$ in the spectrum is a real number, and is in fact an eigenvalue provided that $\lambda \neq \gamma$. Also, it has the above property ii), and iii)' the number of its negative eigenvalues as well as the multiplicity of the zero eigenvalue are finite.

\footnotetext{
${ }^{1}$ Throughout the paper, the eigenvalues are counted according to their multiplicities.
} 
For $\mathbf{Y} \in \mathcal{N}, \mathrm{N}(\mathbf{Y})$ denotes the pair of integers $(z, n)$ where $z$ and $n$ are the multiplicity of the zero eigenvalue and the number of the negative eigenvalues of $\mathbf{Y}$, respectively. We define $\left(z_{1}, n_{1}\right)+\left(z_{2}, n_{2}\right):=\left(z_{1}+z_{2}, n_{1}+n_{2}\right)$, and it readily follows that $\mathrm{N}\left(\left[\begin{array}{ll}\mathbf{Y}_{1} & 0 \\ O & \mathbf{Y}_{2}\end{array}\right]\right)=\mathrm{N}\left(\mathbf{Y}_{1}\right)+\mathrm{N}\left(\mathbf{Y}_{2}\right)$ if $\left[\begin{array}{ll}\mathbf{Y}_{1} & O \\ O & \mathbf{Y}_{2}\end{array}\right] \in \mathcal{N}$. For $\mathbf{Y} \in \mathcal{N}, \mathbf{Y}$ is said to be positive definite [22] (and we denote $\mathbf{Y}>O$ ) if $\langle\mathbf{Y} x, x\rangle>0$ for every nonzero $x \in \mathcal{M}$.

Now, we begin with the following lemma which is an operator version of the Sylvester law of inertia under congruent transformations.

Lemma 1: Suppose that $\mathbf{Y} \in \mathcal{N}$ and that $\mathbf{X}$ is an invertible operator on $\mathcal{M}$. If $\mathbf{X}^{*} \mathbf{Y X} \in \mathcal{N}$, then $\mathrm{N}(\mathbf{Y})=\mathrm{N}\left(\mathbf{X}^{*} \mathbf{Y X}\right)$.

From this lemma, the following lemma is immediate, which is an operator version of the Schur complement arguments.

Lemma 2: Suppose that $\left[\begin{array}{ll}\mathbf{Q} & \mathbf{S} \\ \mathbf{S}^{*} & \mathbf{R}\end{array}\right] \in \mathcal{N}$ and suppose that $\mathbf{Q}$ and $\mathbf{R}$ are invertible when their inverses are referred to. Then

$$
\begin{aligned}
\mathrm{N}\left(\left[\begin{array}{cc}
\mathbf{Q} & \mathbf{S} \\
\mathbf{S}^{*} & \mathbf{R}
\end{array}\right]\right) & =\mathrm{N}\left(\left[\begin{array}{cc}
\mathbf{Q}-\mathbf{S R}^{-1} \mathbf{S}^{*} & O \\
O & \mathbf{R}
\end{array}\right]\right) \\
& =\mathrm{N}\left(\left[\begin{array}{cc}
\mathbf{Q} & O \\
O & \mathbf{R}-\mathbf{S}^{*} \mathbf{Q}^{-1} \mathbf{S}
\end{array}\right]\right) .
\end{aligned}
$$

We also have the following lemmas.

Lemma 3: Suppose $\mathbf{Y} \in \mathcal{N}$. Then, $\mathbf{Y}>O$ if and only if all eigenvalues of $\mathbf{Y}$ are positive [i.e., $\mathrm{N}(\mathbf{Y})=(0,0)$ ].

Lemma 4: Let $X=\left[\begin{array}{cc}O & A \\ A^{*} & O\end{array}\right]$, where $A$ is an $n$-dimensional invertible matrix. Then, $\mathrm{N}(X)=(0, n)$.

The proofs of the above lemmas are given in the Appendix.

\section{BISECTION AlgORITHM FOR COMPUTING THE FREQUENCY RESPONSE GAIN}

\section{A. Frequency Response Gain}

In this section, we first review the notion of the frequency response gain of the sampled-data system shown in Fig. 1 based on the lifting approach [32]. Here, $P$ is the continuous-time plant given by

$$
\begin{aligned}
& \dot{x}(t)=A x(t)+B_{1} w(t)+B_{2} u(t) \\
& z(t)=C_{1} x(t)+D_{11} w(t)+D_{12} u(t) \\
& y(t)=C_{2} x(t)
\end{aligned}
$$

and $\Psi$ is the discrete-time controller given by

$$
\begin{aligned}
\xi[k+1] & =A_{\Psi} \xi[k]+B_{\Psi} \eta[k] \\
\rho[k] & =C_{\Psi} \xi[k]+D_{\Psi} \eta[k] .
\end{aligned}
$$

They are connected with the generalized hold $\mathcal{H}$ with hold function $H(\theta)$ and the ideal sampler $\mathcal{S}$, which work according to the equations

$$
\begin{aligned}
\mathcal{H}: u(k h+\theta) & =H(\theta) \rho[k], \quad(0 \leq \theta<h) \\
\mathcal{S}: \eta[k] & =y(k h)
\end{aligned}
$$

where $h$ is the sampling period. In the following, $\operatorname{dim}(x)$, $\operatorname{dim}(\xi), \operatorname{dim}(u)$ and $\operatorname{dim}(y)$ are denoted by $n_{x}, n_{\xi}, m$ and $l$, respectively.
According to the lifting theory [32], the closed-loop transfer operator of the sampled-data system is given by

$$
\hat{G}(z):=\mathcal{C}(z I-\mathcal{A})^{-1} \mathcal{B}+\mathcal{D}
$$

where

$$
\begin{aligned}
& \mathcal{A}:=\left[\begin{array}{cc}
A_{d}+B_{d 2} D_{\Psi} C_{d 2} & B_{d 2} C_{\Psi} \\
B_{\Psi} C_{d 2} & A_{\Psi}
\end{array}\right] \\
& =\left[\begin{array}{l}
I \\
O
\end{array}\right]\left[\begin{array}{ll}
A_{d} & B_{d 2}
\end{array}\right]\left[\begin{array}{cc}
I & O \\
D_{\Psi} C_{d 2} & C_{\Psi}
\end{array}\right]+\left[\begin{array}{cc}
O & O \\
B_{\Psi} C_{d 2} & A_{\Psi}
\end{array}\right] \\
& \mathcal{B}:=\left[\begin{array}{c}
\mathbf{B}_{1} \\
O
\end{array}\right]=\left[\begin{array}{l}
I \\
O
\end{array}\right] \mathbf{B}_{1} \\
& \mathcal{C}:=\left[\begin{array}{ll}
\mathbf{C}_{1}+\mathbf{D}_{12} D_{\Psi} C_{d 2} & \mathbf{D}_{12} C_{\Psi}
\end{array}\right] \\
& =\left[\begin{array}{ll}
\mathbf{C}_{1} & \mathbf{D}_{12}
\end{array}\right]\left[\begin{array}{cc}
I & O \\
D_{\Psi} C_{d 2} & C_{\Psi}
\end{array}\right] \\
& \mathcal{D}:=\mathbf{D}_{11}
\end{aligned}
$$

with

$$
\begin{aligned}
A_{d} & :=e^{A h} \quad B_{d 2}:=\int_{0}^{h} e^{A(h-\sigma)} B_{2} H(\sigma) d \sigma \\
C_{d 2} & :=C_{2} \\
\mathbf{B}_{1}: w(\cdot) \mapsto & \int_{0}^{h} e^{A(h-\sigma)} B_{1} w(\sigma) d \sigma: L_{2}[0, h) \rightarrow \mathbf{C}^{n_{x}} \\
\mathbf{C}_{1}: x \mapsto & C_{1} e^{A \theta} x: \mathbf{C}^{n_{x}} \rightarrow L_{2}[0, h) \\
\mathbf{D}_{11}: w(\cdot) \mapsto & C_{1} \int_{0}^{\theta} e^{A(\theta-\sigma)} B_{1} w(\sigma) d \sigma+D_{11} w(\theta): \\
& L_{2}[0, h) \rightarrow L_{2}[0, h) \\
\mathbf{D}_{12}: u \mapsto & C_{1} \int_{0}^{\theta} e^{A(\theta-\sigma)} B_{2} H(\sigma) d \sigma u+D_{12} H(\theta) u: \\
& \mathbf{C}^{m} \rightarrow L_{2}[0, h) .
\end{aligned}
$$

The frequency response gain at angular frequency $\varphi$ is defined as the norm of $\hat{G}\left(e^{j \varphi h}\right)$ induced on $L_{2}[0, h)$, i.e., $\left\|\hat{G}\left(e^{j \varphi h}\right)\right\|$ [32]. Without loss of generality, we may confine the range of $\varphi$ to $-\pi / h<\varphi \leq \pi / h$, and we have $\left\|\hat{G}\left(e^{j \varphi h}\right)\right\|=\left\|\hat{G}\left(e^{-j \varphi h}\right)\right\|$. In this paper, we are interested in checking if

$$
\left\|\hat{G}\left(e^{j \varphi h}\right)\right\|<\gamma
$$

for a given number $\gamma$, so that we can obtain a bisection algorithm for the computation of the frequency response gain $\left\|\hat{G}\left(e^{j \varphi h}\right)\right\|$.

Regarding the problem of checking whether or not (15) is true, we may introduce the following assumption without loss of generality.

Assumption $1 D_{11}=O$ : This is because $\left\|\hat{G}\left(e^{j \varphi h}\right)\right\|$ is not smaller than $\left\|D_{11}\right\|$ [32], and, hence, we may always assume $\gamma>\left\|D_{11}\right\|$. Hence, an appropriate $J$-unitary transformation can always reduce the problem of checking (15) to that of checking an inequality of the same form but with $D_{11}=0$ [13]. Assumption 1 guarantees the compactness of the operator $\mathcal{D}$, and, hence, that of $\hat{G}\left(e^{j \varphi h}\right)$ [32]. This enables us to employ the results of the preceding section. Note that $\left\|\hat{G}\left(e^{j \varphi h}\right)\right\|$ is equal to the maximum singular value $\sigma_{1}\left(\hat{G}\left(e^{j \varphi h}\right)\right)$. 


\section{B. Main Results}

Now, we proceed to the topic of deriving a bisection algorithm. In the following, $\zeta$ stands for $e^{j \varphi h}$ for a given value of $\varphi$, and we assume that $\gamma>0$ does not coincide with a singular value of $\mathcal{D}$. Since only 0 is the accumulation point of the singular values of $\mathcal{D}$, this assumption will be satisfied for almost every $\gamma$.

Regarding (15), we can readily show that this condition is equivalent to the following condition by using the Schur complement arguments (Lemma 2).

$$
\left[\begin{array}{cc}
\gamma I & -\hat{G}^{*}(\zeta) \\
-\hat{G}(\zeta) & \gamma I
\end{array}\right]>O
$$

By substituting (5) into (16), the above condition can be restated as $\mathbf{K}(\zeta, \gamma)>O$ where

$$
\begin{aligned}
\mathbf{K}(\zeta, \gamma):= & {\left[\begin{array}{cc}
\gamma I & -\mathcal{D}^{*} \\
-\mathcal{D} & \gamma I
\end{array}\right]-\left[\begin{array}{cc}
\mathcal{B}^{*} & O \\
O & \mathcal{C}
\end{array}\right] } \\
& \times\left[\begin{array}{cc}
O & \zeta I-\mathcal{A} \\
\zeta^{*} I-\mathcal{A}^{*} & O
\end{array}\right]^{-1}\left[\begin{array}{cc}
\mathcal{B} & O \\
O & \mathcal{C}^{*}
\end{array}\right] .
\end{aligned}
$$

Here, note that $\zeta I-\mathcal{A}$ is invertible because $|\zeta|=1$ and the feedback system is assumed to be internally stable and, hence, $\mathcal{A}$ is a stability matrix. By this, together with Lemmas 3 and 4, we can readily see that the condition $\mathbf{K}(\zeta, \gamma)>O$ is equivalent to the condition

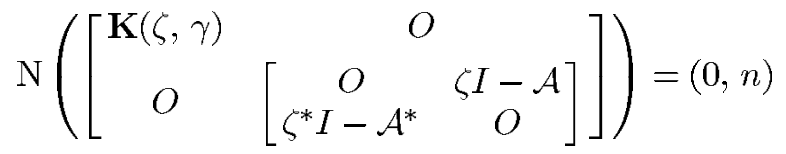

where $n$ denotes the size of the matrix $\mathcal{A}$, i.e., $n=n_{x}+n_{\xi}$. This condition in turn is equivalent to the condition

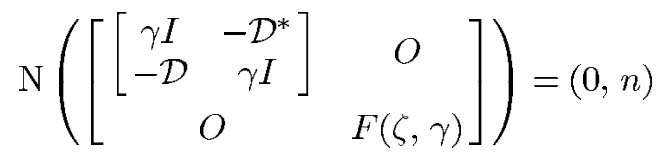

by (17) and Lemma 2, where the finite-dimensional matrix $F(\zeta, \gamma)$ is given by

$$
\begin{aligned}
F(\zeta, \gamma):= & {\left[\begin{array}{cc}
O & \zeta I-\mathcal{A} \\
\zeta^{*} I-\mathcal{A}^{*} & O
\end{array}\right]-\left[\begin{array}{cc}
\mathcal{B} & O \\
O & \mathcal{C}^{*}
\end{array}\right] } \\
\times & {\left[\begin{array}{cc}
\gamma I & -\mathcal{D}^{*} \\
-\mathcal{D} & \gamma I
\end{array}\right]^{-1}\left[\begin{array}{cc}
\mathcal{B}^{*} & O \\
O & \mathcal{C}
\end{array}\right] }
\end{aligned}
$$

Summarizing the above, we can obtain the following theorem.

Theorem 1: Suppose that $\gamma$ is not a singular value of $\mathcal{D}$. Then, the following four statements are equivalent, where $n$ is the size of the square matrix $\mathcal{A}$, and $\nu$ is the number of the singular values of $\mathcal{D}$ larger than $\gamma$.

1)

$$
\|\hat{G}(\zeta)\|<\gamma
$$

2)

$$
\mathrm{N}(\mathbf{K}(\zeta, \gamma))+\mathrm{N}\left(\left[\begin{array}{cc}
O & \zeta I-\mathcal{A} \\
\zeta^{*} I-\mathcal{A}^{*} & O
\end{array}\right]\right)=(0, n)
$$

3)

$$
\mathrm{N}\left(\left[\begin{array}{cc}
\gamma I & -\mathcal{D}^{*} \\
-\mathcal{D} & \gamma I
\end{array}\right]\right)+\mathrm{N}(F(\zeta, \gamma))=(0, n)
$$

4)

$$
\mathrm{N}(F(\zeta, \gamma))=(0, n-\nu)
$$

By Theorem 1, whether or not the condition (15) holds can be checked by counting up the number of the singular values of $\mathcal{D}$ larger than $\gamma$, the number of the negative eigenvalues of the finite-dimensional matrix $F(\zeta, \gamma)$, and the multiplicity of the zero eigenvalue of $F(\zeta, \gamma)$. As for the computation of the first number, we could employ the method of [5] to compute the singular values of $\mathcal{D}$. Alternatively, we can apply the bisection algorithm developed in Section III-D. As for the second and third numbers, the computation of $F(\zeta, \gamma)$ is described in Section III-C. Hence, we can readily obtain a complete bisection algorithm, as opposed to that in [15], from the condition (23).

Before closing this subsection, let us note that Theorem 1 can be extended to such a result that can be used to compute other singular values (not only the maximum singular value) of sampled-data systems via a bisection search. To compute other singular values of sampled-data systems by a bisection search algorithm, it is sufficient to know the open interval $\left(\sigma_{i+1}(\hat{G}(\zeta)), \sigma_{i}(\hat{G}(\zeta))\right)$ in which a given $\gamma$ is included. Now, suppose that $\sigma_{i+1}(\hat{G}(\zeta))<\gamma<\sigma_{i}(\hat{G}(\zeta))$. We can readily see that this condition is equivalent to the condition $\mathrm{N}(\mathbf{K}(\zeta, \gamma))=(0, i)$. Hence, we can readily obtain the following result in a similar way to Theorem 1 .

Theorem 2: Suppose that $\gamma$ is not a singular value of $\mathcal{D}$. Then, the following four statements are equivalent for all nonnegative integers $i$, where $\sigma_{0}(\cdot)$ is defined to be infinity, $n$ is the size of the square matrix $\mathcal{A}$, and $\nu$ is the number of the singular values of $\mathcal{D}$ larger than $\gamma$.

1)

$$
\sigma_{i+1}(\hat{G}(\zeta))<\gamma<\sigma_{i}(\hat{G}(\zeta))
$$

2)

$\mathrm{N}(\mathbf{K}(\zeta, \gamma))+\mathrm{N}\left(\left[\begin{array}{cc}O & \zeta I-\mathcal{A} \\ \zeta^{*} I-\mathcal{A}^{*} & O\end{array}\right]\right)=(0, n+i)$.

3)

$$
\mathrm{N}\left(\left[\begin{array}{cc}
\gamma I & -\mathcal{D}^{*} \\
-\mathcal{D} & \gamma I
\end{array}\right]\right)+\mathrm{N}(F(\zeta, \gamma))=(0, n+i)
$$

4)

$$
\mathrm{N}(F(\zeta, \gamma))=(0, n+i-\nu)
$$

\section{Computation of $F(\zeta, \gamma)$}

In this section, we show how to compute $F(\zeta, \gamma)$ that is used in the bisection algorithm. From (6)-(9), we have

$$
\begin{aligned}
F(\zeta, \gamma)= & {\left[\begin{array}{cc}
O & \zeta I-\tilde{E} \\
\zeta^{*} I-\tilde{E}^{T} & O
\end{array}\right]-\left[\begin{array}{cc}
\tilde{B} & O \\
O & \tilde{C}^{T}
\end{array}\right] } \\
& \times G(\gamma)\left[\begin{array}{cc}
\tilde{B}^{T} & O \\
O & \tilde{C}
\end{array}\right]
\end{aligned}
$$


where

$$
\begin{aligned}
& G(\gamma):=\left[\begin{array}{ccc}
O & A_{d} & B_{d 2} \\
A_{d}^{T} & O & O \\
B_{d 2}^{T} & O & O
\end{array}\right]+\left[\begin{array}{cc}
\mathbf{B}_{1} & O \\
O & \mathbf{C}_{1}^{*} \\
O & \mathbf{D}_{12}^{*}
\end{array}\right] \\
& \times\left[\begin{array}{cc}
\gamma I & -\mathbf{D}_{11}^{*} \\
-\mathbf{D}_{11} & \gamma I
\end{array}\right]^{-1}\left[\begin{array}{ccc}
\mathbf{B}_{1}^{*} & O & O \\
O & \mathbf{C}_{1} & \mathbf{D}_{12}
\end{array}\right] \\
& \tilde{E}:=\left[\begin{array}{cc}
O & O \\
B_{\Psi} C_{d 2} & A_{\Psi}
\end{array}\right], \quad \tilde{B}:=\left[\begin{array}{c}
I \\
O
\end{array}\right] \\
& \tilde{C}:=\left[\begin{array}{cc}
I & O \\
D_{\Psi} C_{d 2} & C_{\Psi}
\end{array}\right] \text {. }
\end{aligned}
$$

When $H(\theta)=C_{H} e^{A_{H} \theta} B_{H}$ is employed as the hold function, the above $G(\gamma)$ can be computed by the formula

$$
\begin{aligned}
G(\gamma)= & {\left[\begin{array}{ccc}
I & 0 & 0 \\
0 & I & 0 \\
0 & 0 & B_{H}^{T} \Gamma_{44}^{-1}
\end{array}\right]\left[\begin{array}{ccc}
\Gamma_{21} & \Gamma_{22} & \Gamma_{23} \\
I & 0 & 0 \\
\Gamma_{41} & \Gamma_{42} & \Gamma_{43}
\end{array}\right] } \\
& \times\left[\begin{array}{ccc}
\Gamma_{11} & \Gamma_{12} & \Gamma_{13} \\
0 & I & 0 \\
0 & 0 & I
\end{array}\right]^{-1}\left[\begin{array}{ccc}
I & 0 & 0 \\
0 & I & 0 \\
0 & 0 & B_{H}
\end{array}\right] .
\end{aligned}
$$

The above equation and (32) at the bottom of the page, give a formula for the computation of $F(\zeta, \gamma)$ via matrix exponentiation. The derivation of the above formula is similar to the arguments in [5], [16], [17], and is omitted.

\section{Bisection Algorithm for Computing the Singular Values of $\mathcal{D}$}

In this section, we show that the singular values of $\mathcal{D}=\mathbf{D}_{11}$ can also be computed via a bisection search. To show this, we employ a technique similar to that used in Theorems 1 and 2, together with the FR-operator representation $P_{11}(j \varphi)$ of the open-loop system from $w$ to $z$. Here, $\underline{P_{11}}(j \varphi)$ is given by

$\underline{P_{11}}(j \varphi)$

$$
=\operatorname{blockdiag}\left[\ldots, P_{11}\left(j \varphi_{-1}\right), P_{11}\left(j \varphi_{0}\right), P_{11}\left(j \varphi_{1}\right), \ldots\right]
$$

where $P_{11}(s)=C_{1}(s I-A)^{-1} B_{1}$ and $\varphi_{m}=\varphi+2 m \pi / h$ [1]. The counterpart to $\underline{P_{11}}(j \varphi)$ in the lifting approach is given by

$$
\mathbf{P}_{11}\left(e^{j \zeta h}\right)=\mathbf{C}_{1}\left(e^{j \varphi h} I-A_{d}\right)^{-1} \mathbf{B}_{1}+\mathbf{D}_{11} .
$$

In [30], it has been shown that the frequency response based on the lifting approach and that based on the FR-operator are isometrically isomorphic. This means that the singular values of $\mathbf{P}_{11}\left(e^{j \varphi h}\right)$ and those of $P_{11}(j \varphi)$ all coincide for each $\varphi$, that is, the following relation holds for each $\varphi$ and every $\gamma>0$.

$$
\begin{aligned}
& \mathrm{N}\left(\left[\begin{array}{cc}
\gamma I & -\mathbf{P}_{11}^{*}\left(e^{j \varphi h}\right) \\
-\mathbf{P}_{11}\left(e^{j \varphi h}\right) & \gamma I
\end{array}\right]\right) \\
& \quad=\mathrm{N}\left(\left[\begin{array}{cc}
\gamma I & -\frac{P_{11}}{\gamma}(j \varphi) \\
-\underline{P_{11}(j \varphi)} & \gamma I
\end{array}\right]\right) .
\end{aligned}
$$

Note that $P_{11}(s)$ is strictly proper by Assumption 1 . This implies that the elements $P_{11}\left(j \varphi_{m}\right)$ tend to zero as $|m|$ goes to infinity. Therefore, we can compute the exact values of all the singular values of $P_{11}(j \varphi)$ larger than $\gamma$ by computing the singular values of the finite-dimensional matrix

$$
\begin{aligned}
& P_{\left.11_{[M]}\right]}(j \varphi) \\
& \quad=\operatorname{blockdiag}\left[P_{11}\left(j \varphi_{-M}\right), \ldots, P_{11}\left(j \varphi_{0}\right), \ldots, P_{11}\left(j \varphi_{M}\right)\right]
\end{aligned}
$$

larger than $\gamma$, if $M$ is sufficiently large. One such $M$ is given as follows, as discussed in [7]. Let

$$
A_{p}=\left[\begin{array}{cc}
-A^{T} & -\frac{1}{\gamma} C_{1}^{T} C_{1} \\
\frac{1}{\gamma} B_{1} B_{1}^{T} & A
\end{array}\right]
$$

and let

$$
\mu=\max \left\{|\lambda|: \lambda \text { is a purely imaginary eigenvalue of } A_{p}\right\} .
$$

Then, it is enough to take $M$ such that $\varphi_{M}>\mu$ and $\varphi_{-M}<$ $-\mu$, which follows from the well-known results of [3]. Hence, it follows that we can obtain the exact value of the right-hand side of (35), and, thus, the left-hand side. On the other hand, using Lemma 2, we can obtain (39) at the bottom of the next page, in a similar way to the arguments in Section III-B, where the finite-dimensional matrix $F_{11}\left(e^{j \varphi h}, \gamma\right)$ is given by

$$
\begin{aligned}
F_{11}\left(e^{j \varphi h}, \gamma\right):= & {\left[\begin{array}{cc}
O & e^{j \varphi h} I \\
e^{-j \varphi h} I & O
\end{array}\right]-G_{11}(\gamma) } \\
G_{11}(\gamma):= & {\left[\begin{array}{cc}
O & A_{d} \\
A_{d}^{T} & O
\end{array}\right]+\left[\begin{array}{cc}
\mathbf{B}_{1} & O \\
O & \mathbf{C}_{1}^{*}
\end{array}\right] } \\
& \times\left[\begin{array}{cc}
\gamma I & -\mathrm{D}_{11}^{*} \\
-\mathrm{D}_{11} & \gamma I
\end{array}\right]^{-1}\left[\begin{array}{cc}
\mathbf{B}_{1}^{*} & O \\
O & \mathbf{C}_{1}
\end{array}\right] \\
= & {\left[\begin{array}{ccc}
I & O & O \\
O & I & O
\end{array}\right] G(\gamma)\left[\begin{array}{cc}
I & O \\
O & I \\
O & O
\end{array}\right] } \\
= & {\left[\begin{array}{cc}
\Gamma_{21} & \Gamma_{22} \\
I & O
\end{array}\right]\left[\begin{array}{cc}
\Gamma_{11} & \Gamma_{12} \\
O & I
\end{array}\right]^{-1} . }
\end{aligned}
$$

$$
\left[\begin{array}{cccc}
\Gamma_{11} & \Gamma_{12} & \Gamma_{13} & 0 \\
\Gamma_{21} & \Gamma_{22} & \Gamma_{23} & 0 \\
0 & 0 & \Gamma_{33} & 0 \\
\Gamma_{41} & \Gamma_{42} & \Gamma_{43} & \Gamma_{44}
\end{array}\right]:=\exp \left(\left[\begin{array}{cccc}
-A^{T} & -\frac{1}{\gamma} C_{1}^{T} C_{1} & -\frac{1}{\gamma} C_{1}^{T} D_{12} C_{H} & 0 \\
\frac{1}{\gamma} B_{1} B_{1}^{T} & A & B_{2} C_{H} & 0 \\
0 & 0 & A_{H} & 0 \\
C_{H}^{T} B_{2}^{T} & \frac{1}{\gamma} C_{H}^{T} D_{12}^{T} C_{1} & \frac{1}{\gamma} C_{H}^{T} D_{12}^{T} D_{12} C_{H} & -A_{H}^{T}
\end{array}\right] h\right) .
$$


Since we already know the value of the left-hand side of (35) as mentioned above, we can compute the value of the left-hand side of (39) by Lemma 4. Therefore, if we focus on the right-hand side of (39), by computing the number of the negative eigenvalues of $F_{11}\left(e^{j \varphi h}, \gamma\right)$, we can obtain the exact number of the negative eigenvalues of the operator $\left[\begin{array}{cc}\gamma I & -\mathbf{D}_{11}^{*} \\ -\mathbf{D}_{11} & \gamma I\end{array}\right]$. Since this is true for each $\gamma>0$, we can compute every singular value of $\mathbf{D}_{11}(=\mathcal{D})$ via a bisection search with respect to $\gamma$. It is sufficient to carry out this bisection search at one fixed angular frequency for which $e^{j \varphi h} I-A_{d}$ is invertible, because $\mathbf{D}_{11}$ is independent of $\varphi$.

\section{RELATED ISSUES AND FuRTHER DEVELOPMENTS}

\section{A. One-Dimensional Search Algorithm}

In this section, we show that another procedure can be obtained from the condition 4) of Theorem 2 for the computation of the frequency response gain and the singular values of $\mathcal{D}$.

Suppose that $\gamma$ is larger than $\|\hat{G}(\zeta)\|$, so that (25) holds for $i=0$. Also suppose that

$$
\sigma_{\nu+1}(\mathcal{D})<\gamma<\sigma_{\nu}(\mathcal{D})
$$

so that there are $\nu$ singular values of $\mathcal{D}$ larger than $\gamma$. Now, as $\gamma$ decreases, the condition (25) fails for $i=0$ eventually when $\gamma$ crosses the value of $\|\hat{G}(\zeta)\|$, and we will have $i>0$ instead. On the other hand, the value of $\nu$ remains unchanged as long as $\gamma$ remains within the range (42), and, hence, by (28), the number of the negative eigenvalues of $F(\zeta, \gamma)$ increases as $\gamma$ crosses $\|\hat{G}(\zeta)\|$. This implies that $\|\hat{G}(\zeta)\|$ can be obtained by searching for the largest value of $\gamma$ across which the number of the negative eigenvalues of $F(\zeta, \gamma)$ increases as $\gamma$ decreases. [In other words, $\|\hat{G}(\zeta)\|$ can be obtained by searching for the largest value of $\gamma$ that satisfies the condition $\operatorname{det}(F(\zeta, \gamma))=0$. See Fig. 2] Fortunately, it readily follows from the structure of $F(\zeta, \gamma)$ as shown in (20) that the eigenvalues of this matrix are uniformly nonincreasing as $\gamma$ decreases, provided that $\gamma$ is within the range of (42) (i.e., provided that $\gamma$ does not cross a singular value of $\mathcal{D}$ ). Therefore, it should be easy to find, in a numerically reliable fashion, the largest value of $\gamma$ across which the number of the negative eigenvalues of $F(\zeta, \gamma)$ increases as $\gamma$ decreases. This gives an alternative method for the computation of the frequency response gain $\|\hat{G}(\zeta)\|$ without a bisection search, which can be carried out without a prior knowledge of the singular values of $\mathcal{D}$.

By the way, suppose that $\gamma$ crosses one of the singular values of $\mathcal{D}$ before it crosses $\|\hat{G}(\zeta)\|$ as $\gamma$ decreases. In this case, the

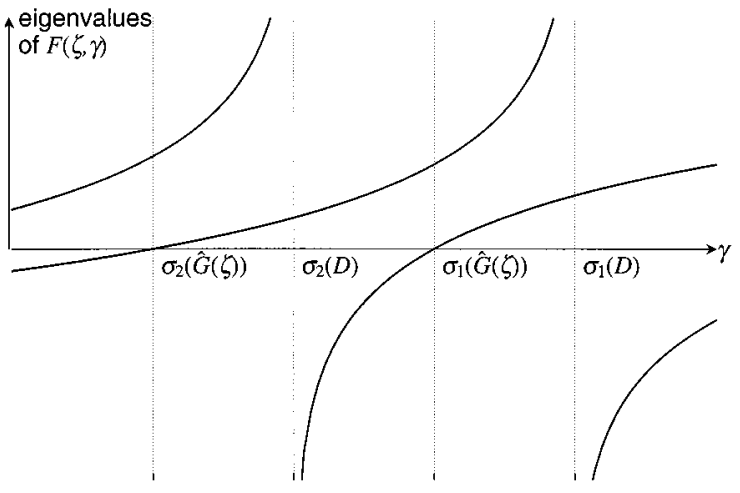

Fig. 2. The behavior of the eigenvalues of $F(\zeta, \gamma)(n=2)$.

inequality (42) fails for the original $\nu$, and this inequality will be satisfied for a larger value of $\nu$. This implies that the right-hand side of (28) must become smaller as $\gamma$ crosses a singular value of $\mathcal{D}$. However, from the above mentioned nonincreasing properties of the eigenvalues of $F(\zeta, \gamma)$ when decreasing $\gamma$, this can happen only by a discontinuous jump of some of the eigenvalues of this matrix at a singular value of $\mathcal{D}$ (see Fig. 2). To put it reverse, the singular values of $\mathcal{D}$ can be found, including their multiplicities, by observing the place and the number of such kind of discontinuous jumps of the eigenvalues of $F(\zeta, \gamma)$. This gives an alternative method for the computation of the singular values of $\mathcal{D}$. For this computation, it is obviously enough to take only one fixed value $\varphi$ (or $\zeta$ ).

The above alternative procedure for the computation of the frequency response gain is in fact closely related to the one given by Yamamoto and Khargonekar [32], which is based on the following proposition (adapted and rearranged to fit our notation).

Proposition 1 (Theorem 2 of [32]): Suppose that $\gamma$ is not a singular value of $\mathcal{D}$. Then, the following two statements are equivalent:

1) $\gamma$ is a singular value of $\hat{G}(\zeta)$;

2) $\operatorname{det}(\zeta \bar{E}(\gamma)-\bar{A}(\gamma))=0$;

where

$$
\begin{aligned}
& \bar{E}(\gamma)=\left[\begin{array}{ccc}
I & -\mathcal{B}\left(\gamma I-\frac{1}{\gamma} \mathcal{D}^{*} \mathcal{D}\right)^{-1} & \mathcal{B}^{*} \\
O & \mathcal{A}^{*}+\mathcal{C}^{*} \mathcal{D}\left(\gamma^{2} I-\mathcal{D}^{*} \mathcal{D}\right)^{-1} \mathcal{B}^{*}
\end{array}\right] \\
& \bar{A}(\gamma)=\left[\begin{array}{ccc}
\mathcal{A}+\mathcal{B}\left(\gamma^{2} I-\mathcal{D} \mathcal{D}^{*} \mathcal{D}\right)^{-1} \mathcal{D}^{*} \mathcal{C} & O \\
-\mathcal{C}^{*}\left(\gamma I-\frac{1}{\gamma} \mathcal{D} \mathcal{D}^{*}\right)^{-1} \mathcal{C} & I
\end{array}\right] .
\end{aligned}
$$

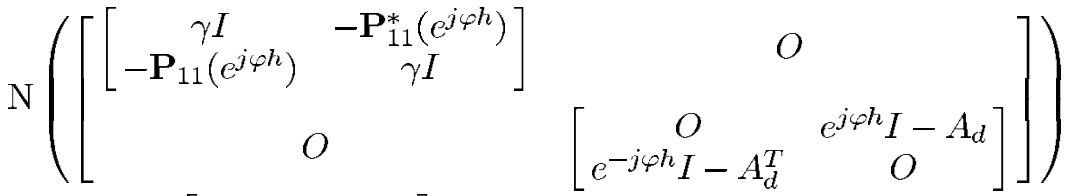

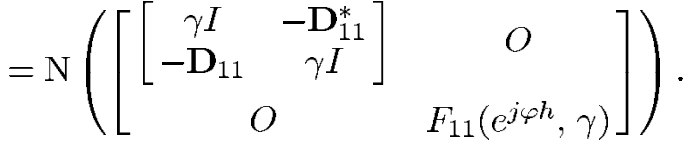


Now, letting $A_{\gamma}:=\mathcal{A}+\mathcal{B}\left(\gamma^{2} I-\mathcal{D}^{*} \mathcal{D}\right)^{-1} \mathcal{D}^{*} \mathcal{C}, B_{\gamma}:=$ $\mathcal{B}\left(\gamma I-(1 / \gamma) \mathcal{D}^{*} \mathcal{D}\right)^{-1} \mathcal{B}^{*}$ and $C_{\gamma}:=\mathcal{C}^{*}\left(\gamma I-(1 / \gamma) \mathcal{D} \mathcal{D}^{*}\right)^{-1} \mathcal{C}$, the condition 2) can be restated as follows.

$$
\operatorname{det}\left(\left[\begin{array}{cc}
\zeta I-A_{\gamma} & -\zeta B_{\gamma} \\
C_{\gamma} & \zeta A_{\gamma}^{*}-I
\end{array}\right]\right)=0 .
$$

On the other hand, our reduced condition given in this subsection is $\operatorname{det}(F(\zeta, \gamma))=0$, or equivalently

$$
\operatorname{det}\left(\left[\begin{array}{cc}
-B_{\gamma} & \zeta I-A_{\gamma} \\
\zeta^{*} I-A_{\gamma}^{*} & -C_{\gamma}
\end{array}\right]\right)=0 .
$$

Naturally, it can easily be verified that (43) and (44) are equivalent. However, the latter has a numerically desirable feature that the involved matrix $F(\zeta, \gamma)$ is self-adjoint (i.e., Hermitian). Furthermore, the eigenvalues of the matrix involved have numerically amenable properties as stated above. Namely, our one-dimensional search has an advantage over that of [32] in that by observing the behavior of all these eigenvalues [rather than only their product, i.e., the determinant in (44)], the frequency response gain $\gamma=\|\hat{G}(\zeta)\|$ can be detected more reliably.

\section{B. Relationship to the Solution of Sampled-Data $H_{\infty}$ Problem}

The exponentiation formula for computing the finite-dimensional matrix $F(\zeta, \gamma)$, or equivalently, that for $G(\gamma)$, frequently appeared also in the existing researches on the $H_{\infty}$ control problem of sampled-data systems [5], [16]. This suggests a strong connection between our study and those existing studies, and we indeed have the following theorem that establishes such a connection.

Theorem 3: Suppose that the discrete-time system $\hat{P}$ given by

$$
\begin{aligned}
\hat{x}[k+1] & =\hat{A} \hat{x}[k]+\hat{B}_{1} \hat{w}[k]+\hat{B}_{2} \rho[k] \\
\hat{z}[k] & =\hat{C}_{1} \hat{x}[k]+\hat{D}_{11} \hat{w}[k]+\hat{D}_{12} \rho[k] \\
\eta[k] & =\hat{C}_{2} \hat{x}[k]
\end{aligned}
$$

satisfies the following three conditions.

1) $G(\gamma)$ given by (30) has the following decomposition:

$$
\begin{aligned}
& {\left[\begin{array}{ccc}
O & A_{d} & B_{d 2} \\
A_{d}^{T} & O & O \\
B_{d 2}^{T} & O & O
\end{array}\right]+\left[\begin{array}{cc}
\mathbf{B}_{1} & O \\
O & \mathbf{C}_{1}^{*} \\
O & \mathrm{D}_{12}^{*}
\end{array}\right]} \\
& \times\left[\begin{array}{cc}
\gamma I & -\mathbf{D}_{11}^{*} \\
-\mathbf{D}_{11} & \gamma I
\end{array}\right]^{-1}\left[\begin{array}{ccc}
\mathbf{B}_{1}^{*} & O & O \\
O & \mathbf{C}_{1} & \mathbf{D}_{12}
\end{array}\right] \\
& =\left[\begin{array}{ccc}
O & \hat{A} & \hat{B}_{2} \\
\hat{A}^{T} & O & O \\
\hat{B}_{2}^{T} & O & O
\end{array}\right]+\left[\begin{array}{cc}
\hat{B}_{1} & O \\
O & \hat{C}_{1}^{T} \\
O & \hat{D}_{12}^{T}
\end{array}\right] \\
& \times\left[\begin{array}{cc}
\gamma I & -\hat{D}_{11}^{T} \\
-\hat{D}_{11} & \gamma I
\end{array}\right]^{-1}\left[\begin{array}{ccc}
\hat{B}_{1}^{T} & O & O \\
O & \hat{C}_{1} & \hat{D}_{12}
\end{array}\right] .
\end{aligned}
$$

2) $\gamma>\sigma_{1}\left(\hat{D}_{11}\right)$
3) For all $A_{\Psi}, B_{\Psi}, C_{\Psi}$ and $D_{\Psi}$, the matrix $\mathcal{A}$ is stable if and only if the matrix $\hat{\mathcal{A}}$ is, where

$$
\begin{aligned}
& \mathcal{A}=\left[\begin{array}{cc}
A_{d}+B_{d 2} D_{\Psi} C_{d 2} & B_{d 2} C_{\Psi} \\
B_{\Psi} C_{d 2} & A_{\Psi}
\end{array}\right] \\
& \hat{\mathcal{A}}=\left[\begin{array}{cc}
\hat{A}+\hat{B}_{2} D_{\Psi} \hat{C}_{2} & \hat{B}_{2} C_{\Psi} \\
B_{\Psi} \hat{C}_{2} & A_{\Psi}
\end{array}\right] .
\end{aligned}
$$

Then, $\hat{P}$ can be used as an equivalent discrete-time system for solving the $H_{\infty}$ control problem of the original sampled-data system.

Theorem 3 can be proved by replacing $G(\gamma)$ in (29) with the right-hand side of (46), and tracing the arguments in Section III-B in the reversed way mutatis mutandis. The importance of Theorem 3 lies in that it provides a general class of discrete-time systems that can be used for solving the $H_{\infty}$ problem of sampled-data systems. Indeed, we can verify that both the discrete-time system given by [5] (which we call the B-P type realization) and that by [16] (H-H-Y type) are special examples of $\hat{P}$ satisfying the conditions of the above theorem; we can interpret the B-P type realization as the one obtained by solving (46) for $\hat{P}$ under the constraint $\hat{D}_{11}=0$, while the $\mathrm{H}-\mathrm{H}-\mathrm{Y}$ type under the constraint $\hat{A}=A_{d}$ and $\hat{B}_{2}=B_{d 2}$.

As for the above theorem, it is quite important to note not only its assertion itself but also the following fact: essentially the same arguments as in the computation of the frequency response gain-i.e., the infinite-dimensional congruent transformation techniques developed in this paper-have been applied successfully also to the $H_{\infty}$ control problem. It is precisely this point that distinguishes our approach to the $H_{\infty}$ sampled-data control problem from the conventional approach; the latter is mostly based on the loop-shifting technique [5], [16], which is suitable only to the $H_{\infty}$ control problem and cannot be applied to the computation of the frequency response gain, in general. In other words, the conventional approach with the loop-shifting techniques cannot provide a unified framework to the two problems, in spite of their superficial close relationship. As described in the Introduction, this is because the loop-shifting technique is based on the positivity of a certain operator, and, thus, we need to assume $\gamma>\|\mathcal{D}\|$ to apply this technique; even though this assumption causes no loss of generality in the $H_{\infty}$ control problem, the frequency response gain can be smaller than $\|\mathcal{D}\|$, so that we may not assume $\gamma>\|\mathcal{D}\|$ in the computation of the frequency response gain. This has been precisely the major obstacle in deriving a complete bisection algorithm for the computation of the frequency response gain, and, hence, in establishing a unified framework for the two related problems with the loop-shifting approach.

\section{Bisection Algorithm Based on the FR-Operator}

In this section, we give an alternative bisection algorithm for computing the frequency response gain of sampled-data systems, which is based on the FR-operator. The FR-operator counterpart to $\hat{G}\left(e^{j \varphi h}\right)$ is $\underline{G}(j \varphi)$ [11], which is given by

$$
\begin{aligned}
\underline{G}(j \varphi)= & \underline{P_{11}}(j \varphi)+\frac{1}{h} \underline{P_{12} H}(j \varphi) \Psi\left(e^{j \varphi h}\right) \\
& \times\left(I-\Pi_{22}\left(e^{j \varphi h}\right) \Psi\left(e^{j \varphi h}\right)\right)^{-1} \underline{P_{21}}(j \varphi)
\end{aligned}
$$


where

$$
\begin{aligned}
& \underline{P_{12} H}(j \varphi)=\left[\begin{array}{c}
\vdots \\
P_{12}\left(j \varphi_{-1}\right) \hat{H}\left(j \varphi_{-1}\right) \\
P_{12}\left(j \varphi_{0}\right) \hat{H}\left(j \varphi_{0}\right) \\
P_{12}\left(j \varphi_{1}\right) \hat{H}\left(j \varphi_{1}\right) \\
\vdots \\
P_{21}
\end{array}\right] \\
&
\end{aligned}
$$

Here, $P_{i j}(s)=C_{i}(s I-A)^{-1} B_{j}+D_{i j}, \Pi_{22}(z)$ is the pulse transfer function of $\mathcal{S} P_{22}(s) \mathcal{H}$, and $\hat{H}(s)$ is the transfer function of the hold device. Note that the way an inverse appears in (48) and that in (5) are parallel. Also note that Assumption 1 guarantees that $P_{11}(j \varphi)$ is compact, and so is $\underline{G}(j \varphi)$. Therefore, it is possible to derive an FR-operator counterpart to Theorem 1 in a similar way to the preceding section.

Theorem 4: Suppose that $\gamma$ is not a singular value of $P_{11}(j \varphi)$. Then, the following four statements are equivalent, where $l$ is the number of the outputs of $P_{22}(s)$, and $\underline{\nu}$ the number of the singular values of $P_{11}(j \varphi)$ larger than $\gamma$.

1)

$$
\|\underline{G}(j \varphi)\|<\gamma
$$

2)

$$
\begin{aligned}
& \mathrm{N}(\underline{K}(\varphi, \gamma))+\mathrm{N} \\
& \quad \times\left(\left[\begin{array}{cc}
O & I-\Pi_{22}\left(e^{j \varphi h}\right) \Psi\left(e^{j \varphi h}\right)
\end{array}\right]\right) \\
& =(0, l) ;
\end{aligned}
$$

3)

$$
\mathrm{N}\left(\left[\begin{array}{cc}
\gamma I & -\underline{P_{11}^{*}}(j \varphi) \\
-\underline{P_{11}}(j \varphi) & \gamma I
\end{array}\right]\right)+\mathrm{N}(\underline{F}(\varphi, \gamma))=(0, l)
$$

4)

$$
\mathrm{N}(\underline{F}(\varphi, \gamma))=(0, l-\underline{\nu})
$$

where

$$
\begin{aligned}
\underline{K}(\varphi, & \gamma) \\
:= & {\left[\begin{array}{cc}
\gamma I & -P_{11}{ }^{*}(j \varphi) \\
-\underline{P_{11}}(j \varphi) & \gamma I
\end{array}\right] } \\
& -\frac{1}{h}\left[\begin{array}{cc}
\underline{P}_{21}^{*}(j \varphi) & O \\
O & \frac{P_{12} H(j \varphi) \Psi\left(e^{j \varphi h}\right)}{]}
\end{array}\right] \\
& \times\left[\begin{array}{cc}
I-\Psi_{22}^{*}\left(e^{j \varphi h}\right) \Psi\left(e^{j \varphi h}\right) \\
I \Pi_{22}^{*}\left(e^{j \varphi h}\right)
\end{array}\right] \\
& \times\left[\begin{array}{cc}
\frac{P_{21}(j \varphi)}{O} & O \\
O & \Psi^{*}\left(e^{j \varphi h}\right) \underline{P}_{12} H^{*}(j \varphi)
\end{array}\right]^{-1}
\end{aligned}
$$

$$
\begin{aligned}
& \underline{F}(\varphi, \gamma) \\
& :=\left[\begin{array}{cc}
O & I-\Pi_{22}\left(e^{j \varphi h}\right) \Psi\left(e^{j \varphi h}\right) \\
I-\Psi^{*}\left(e^{j \varphi h}\right) \Pi_{22}^{*}\left(e^{j \varphi h}\right) & O
\end{array}\right] \\
& -\frac{1}{h}\left[\begin{array}{cc}
\underline{P_{21}}(j \varphi) & O \\
O & \Psi^{*}\left(e^{j \varphi h}\right) \underline{P_{12} H^{*}}(j \varphi)
\end{array}\right]
\end{aligned}
$$

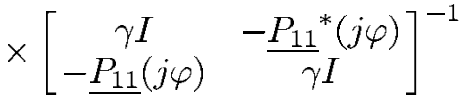

$$
\begin{aligned}
& \times\left[\begin{array}{cc}
{\underline{P_{21}}}^{*}(j \varphi) & O \\
O & \underline{P_{12} H}(j \varphi) \Psi\left(e^{j \varphi h}\right)
\end{array}\right] .
\end{aligned}
$$

Remark 1: In Theorem 4, the size of the square matrix $\underline{F}(\varphi, \gamma)$ is twice the number of the outputs of $P_{22}(s)$. The dual form to Theorem 4, in which the size of $\underline{F}(\varphi, \gamma)$ is twice the number of the inputs of $P_{22}(s)$, can be obtained in a similar way.

From Theorem 4, we can check the condition (49) by (51), so that we can readily obtain a bisection algorithm for the computation of $\|\underline{G}(j \varphi)\|\left(=\left\|\hat{G}\left(e^{j \varphi h}\right)\right\|\right)$. The computation of the first term on the left-hand side of (51) has already been explained. Here, we show how to compute $\underline{F}(\varphi, \gamma)$. Observe that (54) can be rewritten as

$$
\begin{aligned}
& \underline{F}(\varphi, \gamma)=\left[\begin{array}{cc}
O & I \\
I & O
\end{array}\right]-\left[\begin{array}{cc}
I & O \\
O & \Psi^{*}\left(e^{j \varphi h}\right)
\end{array}\right] \\
& \times \Phi_{\gamma}\left(e^{j \varphi h}\right)\left[\begin{array}{cc}
I & O \\
O & \Psi\left(e^{j \varphi h}\right)
\end{array}\right]
\end{aligned}
$$

where

$$
\begin{aligned}
\Phi_{\gamma}\left(e^{j \varphi h}\right):= & {\left[\begin{array}{cc}
O & \Pi_{22}\left(e^{j \varphi h}\right) \\
\Pi_{22}^{*}\left(e^{j \varphi h}\right) & O
\end{array}\right] } \\
& +\frac{1}{h}\left[\begin{array}{cc}
\underline{P_{21}}(j \varphi) & O \\
O & \frac{P_{12} H^{*}}{}(j \varphi)
\end{array}\right] \\
& \times\left[\begin{array}{cc}
\gamma I & -\underline{P}_{11}^{*}(j \varphi) \\
-P_{11}(j \varphi) & { }^{-1}
\end{array}\right] \\
& \times\left[\begin{array}{cc}
\underline{P_{21}}(j \varphi) & O \\
O & \underline{P_{12} H}(j \varphi)
\end{array}\right]
\end{aligned}
$$

It can be shown that $\Phi_{\gamma}\left(e^{j \varphi h}\right)$ is the frequency pulse-transfer function of the discrete-time system given by

$$
\Phi_{\gamma}(z):=\left[\begin{array}{c|cc}
\Phi_{\gamma 11} & -\Phi_{\gamma 11}\left[\begin{array}{c}
C_{2}^{T} \\
O
\end{array}\right] & \Phi_{\gamma 12} \\
\hline\left[\begin{array}{ll}
O & C_{2}
\end{array}\right] & O & O \\
\Phi_{\gamma 21} & -\Phi_{\gamma 21}\left[\begin{array}{c}
C_{2}^{T} \\
O
\end{array}\right] & \Phi_{\gamma 22}
\end{array}\right]
$$

where

$$
\begin{gathered}
{\left[\begin{array}{c:c}
\Phi_{\gamma 11} & \Phi_{\gamma 12} \\
-\Phi_{\gamma 21} & \Phi_{\gamma 22}
\end{array}\right]} \\
{\left[\begin{array}{ccc}
\Gamma_{11} & \Gamma_{12} & \Gamma_{13} \\
\hdashline \Gamma_{41} & \Gamma_{41} & \Gamma_{43}
\end{array}\right](58)}
\end{gathered}
$$

and $\Gamma_{i j}$ are given by (32). The derivation of (57) and (58) is similar to those arguments used in [17], and is omitted. See [17] for details. 
It would be interesting to observe that the resulting finite-dimensional matrix $\underline{F}(\varphi, \gamma)$ differs from the finite-dimensional matrix $F\left(e^{j \varphi h}, \gamma\right)$ that we reached in the lifting approach. Comparing both approaches, we can see the following.

- When we use the lifting-based method, it is sufficient to compute $\nu$ at one angular frequency, since the infinite-dimensional operator $\mathcal{D}$ is independent of $\varphi$. On the other hand, when we use the FR-operator-based method, we have to compute $\underline{\nu}$ for all angular frequencies at which the frequency response gain is computed, since the infinite-dimensional operator $P_{11}(j \varphi)$ depends on $\varphi$. Therefore, concerning the computational load for the infinite-dimensional part, the lifting-based method has an advantage over the FR-operator-based method.

- Regarding the size of the finite-dimensional matrix, the size of $F\left(e^{j \varphi h}, \gamma\right)$, corresponding to the lifting approach, is $2 n$ where $n$ is the sum of the order of the continuous-time generalized plant and that of the discrete-time controller. On the other hand, the size of $F(\varphi, \gamma)$, corresponding to the FR-operator approach, is $2 l$ or $2 m$ where $l$ and $m$ are the numbers of the rows and columns of $P_{22}(s)$, respectively. Usually, $l$ or $m$ is less than $n$. Also, both $F\left(e^{j \varphi h}, \gamma\right)$ and $\underline{F}(\varphi, \gamma)$ can be computed from the same exponentiation (32). Therefore, concerning the computational load for the finite-dimensional part, the FR-operator-based method has an advantage over the lifting-based method.

\section{Possible Applications of the Properties of the Congruent Transformation}

As seen in the preceding sections, the properties of the congruent transformation are quite useful for the computation of the frequency response gain of sampled-data systems, and also for the solution of the sampled-data $H_{\infty}$ control problem. As a matter of fact, the congruent transformation approach developed in this paper can be applied to a wide class of problems on systems and control. For example, applying a similar technique, the well-known bisection algorithm for computing the $H_{\infty}$ norm of a continuous-time system [3] can be derived quite elementarily (i.e., purely algebraically, without resorting to the system-theoretic notion such as inverse systems as in [3]).

This technique can also be applied to check, e.g., the quadratic constraints for sampled-data systems. Here, we consider the condition

$$
\begin{aligned}
& {\left[\begin{array}{c}
(\zeta I-\mathcal{A})^{-1} \mathcal{B} \\
I
\end{array}\right]^{*}\left[\begin{array}{ll}
M_{11} & M_{12} \\
M_{12}^{*} & M_{22}
\end{array}\right]\left[\begin{array}{c}
(\zeta I-\mathcal{A})^{-1} \mathcal{B} \\
I
\end{array}\right]<O} \\
& (\forall \zeta \text { s.t. }|\zeta|=1)
\end{aligned}
$$

where $\mathcal{A}$ is a stability matrix, and $M_{11}, M_{12}$, and $-M_{22}$ are, respectively, an Hermitian matrix, a compact operator, and an invertible operator belonging to $\mathcal{N}$, and they depend on the operators $\mathcal{C}$ and $\mathcal{D}$, in general. Such a quadratic constraint corresponds to the one regarding the Kalman-Yakubovich-Popov lemma in the continuous or discrete-time case [24]. Now, it can readily be seen that (59) is restated as follows:

$-M_{22}-\left[\begin{array}{ll}\mathcal{B}^{*} & M_{12}^{*}\end{array}\right]\left[\begin{array}{cc}O & \zeta I-\mathcal{A} \\ \zeta^{*} I-\mathcal{A}^{*} & -M_{11}\end{array}\right]^{-1}\left[\begin{array}{c}\mathcal{B} \\ M_{12}\end{array}\right]>O$.

Note that the inverse in (60), which we denote here by $R^{-1}$, is a finite-dimensional matrix, and we can show $\mathrm{N}(R)=(0, n)$, where $n$ is the size of the matrix $\mathcal{A}$. Also note that $-M_{22} \in \mathcal{N}$ by assumption. Therefore, again we can apply a technique similar to that in the preceding sections for reducing the test of the condition (60) to a finite-dimensional test. A similar treatment is also suggested in [10] in the context of the study of positive real sampled-data systems [26], [27]. These suggest useful applications of the congruent transformation approach to the IQC method for various problems on systems and control [21].

\section{NUMERICAL STUDY}

In this section, we apply the two bisection algorithms and the one-dimensional (1-D) search algorithm given in the preceding sections to compute the frequency response gain and singular values of sampled-data systems.

\section{A. Comparison Between the Lifting-Based Method and the FR-Operator-Based Method}

Here, we compare the bisection algorithms based on the lifting approach and that based on the FR-operator approach from the viewpoint of the computational load to compute the singular value plot of a sampled-data system.

Example 1: Consider the continuous-time plant $G(s)$ and the continuous-time controller $C_{r}(s)$ given by

$$
\begin{gathered}
G(s)=\frac{1}{4 s^{2}} \cdot \frac{(s / a+1) \prod_{i=0}^{1}\left\{\left(s / \omega_{i}\right)^{2}+2 \zeta_{i}\left(s / \omega_{i}\right)+1\right\}}{\prod_{i=2}^{4}\left\{\left(s / \omega_{i}\right)^{2}+2 \zeta_{i}\left(s / \omega_{i}\right)+1\right\}} \\
C_{r}(s)=\frac{0.0513 s^{3}+0.00424 s^{2}+0.0296 s+0.00157}{s^{4}+0.693 s^{3}+0.779 s^{2}+0.293 s+0.0739}
\end{gathered}
$$

where $a=4.84, \zeta_{0}=0.02, \zeta_{1}=-0.4, \zeta_{2}=\zeta_{3}=\zeta_{4}=0.02$, $\omega_{0}=1, \omega_{1}=5.65, \omega_{2}=0.765, \omega_{3}=1.41, \omega_{4}=1.85$ [2]. We compute the singular value plot of the following sampled-data system.

$P(s): \quad P_{11}(s)=P_{12}(s)=G(s), P_{21}(s)=P_{22}(s)=$ $-G(s)$.

$\Phi(z)$ : $\quad$ discretization of $C_{r}(s)$ by the Tustin (bilinear) transformation with sampling period $h=8$.

Here, based on Theorem 2, we compute seven singular values from the largest for each angular frequency, where the number of the angular frequencies is 200 . To compute the initial upper and lower bounds of the singular values, we use the method given by [14]. The CPU used for the computation is Pentium II $300 \mathrm{MHz}$. The singular value plot of this example that is accurate up to the tolerance of $0.01[\mathrm{~dB}]$ (which we can guarantee by employing a dB-based bisection method) is shown in Fig. 3, and the computational load (CPU-time) is shown in Table I. In this table, $T_{\text {inf }}$ denotes the CPU-time for computing the singular 


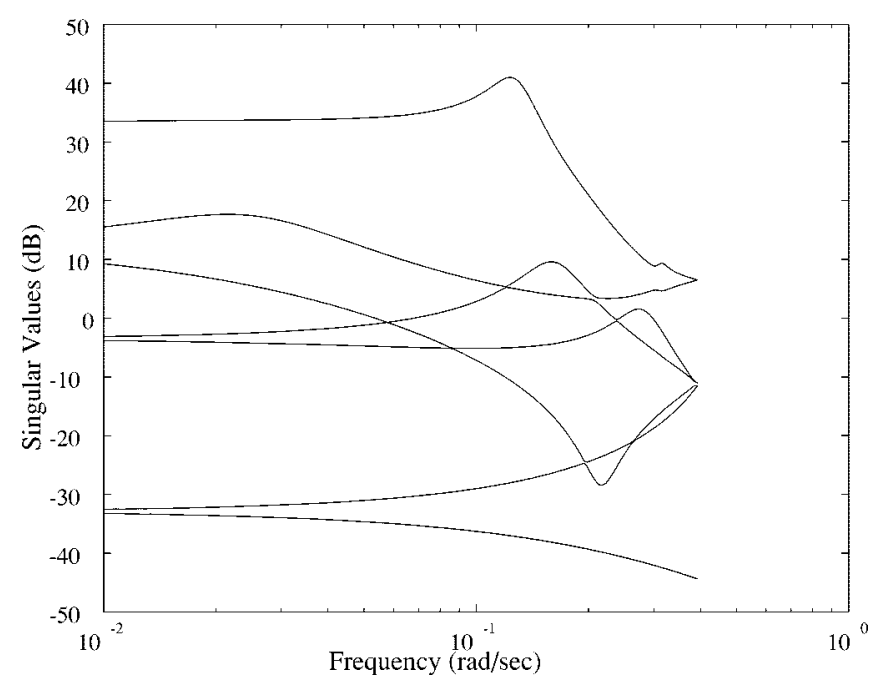

Fig. 3. Singular value plot of Example 1.

TABLE I

COMPUTATIONAL LOAD FOR THE BISECTION SEARCH (CPU-TIME [sec])

\begin{tabular}{|c|c|c|c|}
\hline & \multicolumn{2}{|c|}{ CPU-time } & \multirow{2}{*}{$\begin{array}{l}\text { number of } \\
\gamma \text {-iterations }\end{array}$} \\
\hline & Lifting & FR-Op. & \\
\hline$\overline{T_{\text {inf }}}$ & 1.16 & 2.45 & \\
\hline$T_{1}$ & 0 & 0 & $0^{*}$ \\
\hline$T_{2}$ & 0 & 0 & $0^{*}$ \\
\hline$T_{3}$ & 0.35 & 0.23 & 13 \\
\hline$T_{4}$ & 0.55 & 0.34 & 21 \\
\hline$T_{5}$ & 7.23 & 3.97 & 310 \\
\hline$T_{6}$ & 36.81 & 20.50 & 1579 \\
\hline$T_{7}$ & 45.25 & 24.75 & 1891 \\
\hline
\end{tabular}

*The initial upper and lower bounds for the first and second singular values were close enough, so that no bisection loop was run.

values of the infinite-dimensional operator $\mathcal{D}$ or $P_{11}(j \varphi)$, and $T_{i}$ denotes the one for computing, with the bisection algorithms, the $i$ th largest singular value for all frequencies. For reference, within the range of Fig. 3, there are singular values of $\mathcal{D}$ at about $13.17,-1.2,-6.6,-12.7,-23.9,-34.7$, and $-44.2[\mathrm{~dB}]$.

Regarding $T_{\text {inf }}$, the lifting-based method is superior to the FR-operator-based method. This is because the lifting-based method computes the singular values of the infinite-dimensional operator $\mathcal{D}$ from the value of $P_{11}(j \varphi)$ only at one fixed frequency $\varphi$ (see Section III-D), although the FR-operator-based method needs the singular values of $P_{11}(j \varphi)$ for all frequencies $\varphi$ as stated in Section IV-C. On the contrary, regarding the CPU-times $T_{i}$ for the main body of the bisection algorithm, the FR-operator-based method is superior to the lifting-based method. This is because the size of the finite-dimensional matrix $\underline{F}(\varphi, \gamma)$ corresponding to the FR-operator-based method, is smaller than that of $F\left(e^{j \varphi h}, \gamma\right)$ corresponding to the liftingbased method. Indeed, in this example, the size of $\underline{F}(\varphi, \gamma)$ is two, and the size of $F\left(e^{j \varphi h}, \gamma\right)$ is 24 . Table I shows that the effect of the size of the finite-dimensional matrix becomes significant when the number of iteration increases.

Summarizing the above, we can conclude that when the difference between the initial upper bound and lower bound is large, the FR-operator-based method is superior to the liftingbased method since the computational load for the main body

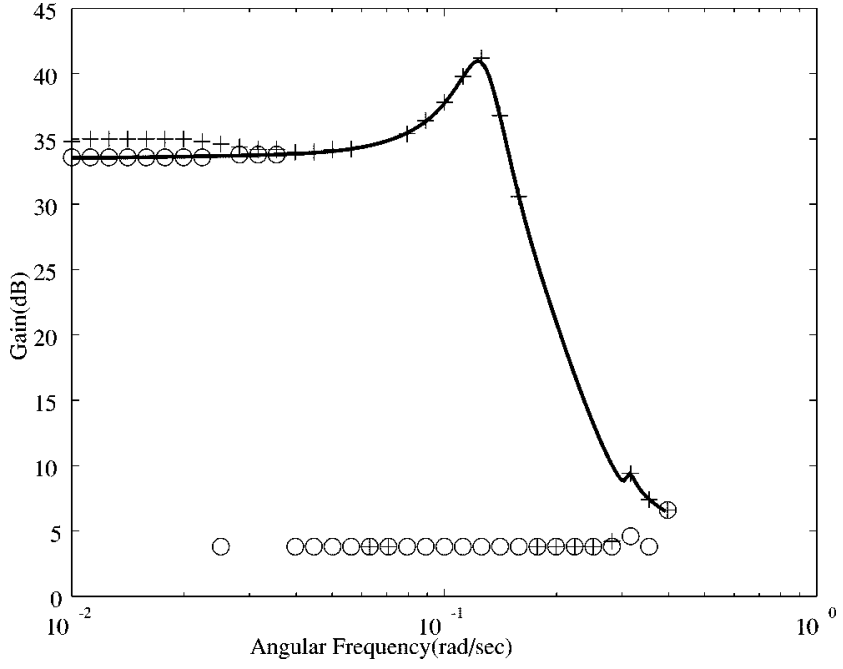

Fig. 4. $\Delta=0.2, e=0.01,0.001$.

of the bisection algorithm (i.e., the part of the finite-dimensional matrix computation) becomes dominant and since the FR-operator-based approach deals with a smaller size of matrix. On the contrary, when the difference between the initial upper bound and lower bound is small, the lifting-based method is superior to the FR-operator-based method since the computational load for the preparation part of the bisection algorithm (i.e., the part of computing the singular values of infinite-dimensional operators) becomes dominant and since the lifting-based approach requires such computation only once.

\section{B. Comparison with the Method of Yamamoto and Khargonekar}

Here, we compute the frequency response gain of the sampled-data system given in Example 1 with the method by Yamamoto and Khargonekar [32]. More precisely, we use the condition (44) instead of (43) for better numerical properties. For simplicity, we decrease $\gamma$ from $44[\mathrm{~dB}]$ to $4[\mathrm{~dB}]$ with a step of $\Delta[\mathrm{dB}]$, since we know from Fig. 3 that the frequency response gain plot is actually within this range. The one-dimensional search is carried out at 33 angular frequencies, and, for each frequency, if the magnitude of the determinant (44) becomes less than $e$ as we decrease $\gamma$, then the frequency response gain is regarded as equal to $\gamma$, where we take $e=0.01$ or 0.001 . Figs. 4 and 5 show the results for $\Delta=0.2$ and for $\Delta=0.002$, respectively, where "+" and "o" denote the plots for $e=0.01$ and $e=0.001$, respectively. Even though we refer to the computational load shown in Table II since it is surely an important factor that may not be neglected, we stress that our primary intention here is to highlight the numerical reliability of our bisection algorithm.

From Figs. 4 and 5, we can observe that we need to take a sufficiently small $e$ to get accurate results, and we also need to take a correspondingly small $\Delta$ as we make $e$ smaller. However, from Table II, we can see that we need a very long time if we make both $e$ and $\Delta$ small. How seriously this computation time gets longer entirely depends on how good the initial upper and lower bounds are, but it is generally true that this one-dimensional search is time-consuming compared with the bisection 


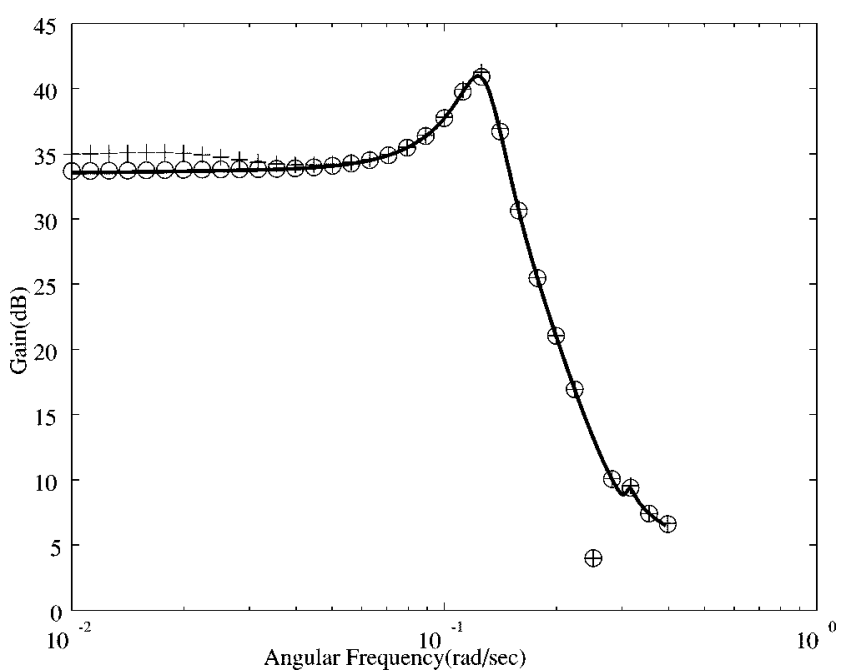

Fig. 5. $\Delta=0.002, e=0.01,0.001$.

TABLE II

COMPUTATIONAL LOAD FOR THE ONE-DimENSIONAL SEARCH (CPU-TIME [sec])

\begin{tabular}{c|c|c|c}
\hline \multicolumn{2}{c|}{} & \multicolumn{2}{c}{$\Delta[\mathrm{dB}]$} \\
\cline { 3 - 4 } \multicolumn{2}{c}{} & 0.2 & 0.002 \\
\hline$e$ & 0.01 & 25.14 & 1958.23 \\
\cline { 2 - 4 } & 0.001 & 42.35 & 2001.20 \\
\hline
\end{tabular}

algorithm. Indeed, for the same setting (i.e., the computation is carried out at 33 angular frequencies starting from the uniform upper bound $44 \mathrm{~dB}$ and lower bound $4 \mathrm{~dB}$, and is accurate up to the tolerance of $0.002 \mathrm{~dB}$ ), the bisection search takes only 9.17 [sec]. Another important thing that we must focus on in Fig. 5 is that the computation of the frequency response gain at $\varphi=10^{-0.6} \approx 0.25[\mathrm{rad} / \mathrm{sec}]$ is quite hard with this 1-D search. The situation does not change so much by making $e$ and $\Delta$ small. This can be seen from Fig. 6, where the dependence of the determinant of (44) versus $\gamma$ is plotted for this frequency. Since the slope of this curve at the zero-crossing point is very steep, it is hard to detect that point by a discrete search on $\gamma$. Note that the slope there is steep because that point (i.e., the frequency response gain at this frequency) is close to the discontinuous point of the plot (namely, a singular value of $\mathcal{D}$-recall the arguments of Section IV-A and Fig. 2). This implies that it is generally hard to compute, with this one-dimensional search algorithm, the frequency response gain at those frequencies where the frequency response gain is close to a singular value of $\mathcal{D}$.

\section{CONCLUSION}

In this paper, we gave a complete bisection algorithm for computing the frequency response gain of sampled-data systems. To be more precise, we derived two different methods, one with the lifting approach and the other with the FR-operator approach, but with the same technique. Namely, they are both based on the properties of the infinite-dimensional congruent transformation (i.e., the Schur complement arguments and the Sylvester law of inertia), and we focused on how to count up the numbers of the negative eigenvalues of self-adjoint operators. Furthermore, since the algorithm with the lifting ap-

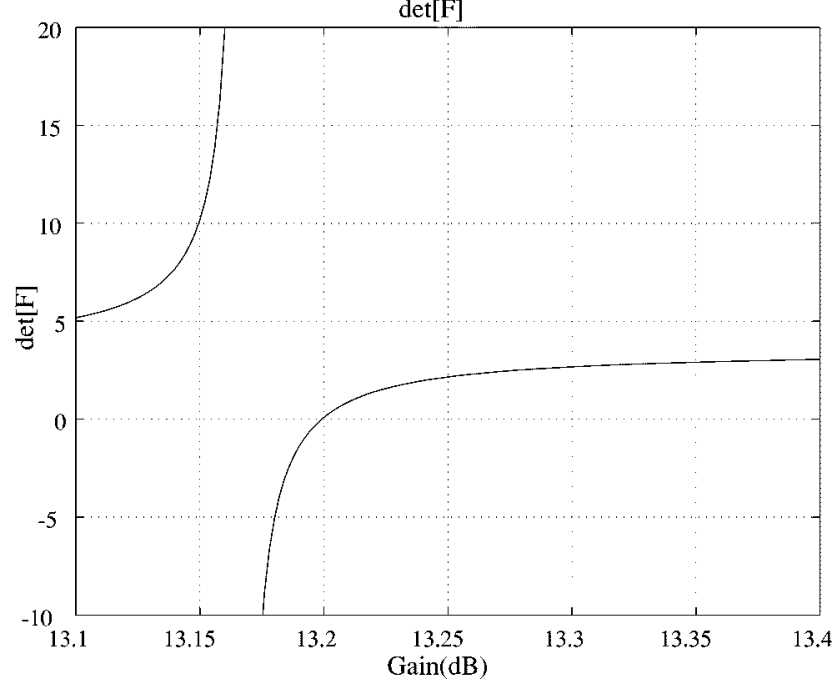

Fig. 6. $\gamma$ vs. $\operatorname{det}\left(F\left(e^{j \varphi h}, \gamma\right)\right)$ plot at $\varphi=10^{-0.6}$

proach requires to compute the singular values of the operator $\mathcal{D}$, we gave a bisection algorithm for their computation as well, again with the same technique. All these algorithms can be carried out easily with the state-space matrices of the sampled-data system, and the effectiveness of the derived bisection algorithms is demonstrated through a numerical example.

Also, some important related issues were discussed such as the relationship to the existing 1-D search method [32]. Furthermore, the link between computing the frequency response gain and solving the sampled-data $H_{\infty}$ problem was made clearer. This contrasts with the apparently prevailing belief (see, e.g., [15]) that the former is essentially much harder than the latter, which comes from some difficulties stemming from the fact that the frequency response gain can be smaller than $\|\mathcal{D}\|$ while the $H_{\infty}$-norm is no smaller than $\|\mathcal{D}\|$.

\section{APPENDIX PROOFS OF LEMMAS}

Proof of Lemma 1: Let $\mathbf{Y}=\gamma I-\mathbf{Z}$ where $\gamma>0$ and $\mathbf{Z} \in \mathcal{W}$. The spectral decomposition theorem assures that there exists a complete orthonormal set $\left\{e_{1}, e_{2}, \ldots\right\}$ consisting of the eigenvectors of $\mathbf{Z}$, such that for any $x \in \mathcal{M}, \mathbf{Z} x$ can be expressed as

$$
\mathbf{Z} x=\sum_{i=1}^{\infty} \lambda_{i}\left\langle x, e_{i}\right\rangle e_{i}
$$

where $\lambda_{i}$ is the eigenvalue of $\mathbf{Z}$ corresponding to $e_{i}$ [34, Corollary 8.16]. We first show that the above spectral decomposition can be extended to $\mathbf{Y} \in \mathcal{N}$. To this end, for $x \in \mathcal{M}$, let us define $\mathbf{Y}_{n} x=\sum_{i=1}^{n}\left(\gamma-\lambda_{i}\right)\left\langle x, e_{i}\right\rangle e_{i}$, and show $\mathbf{Y} x=$ $\lim _{n \rightarrow \infty} \mathbf{Y}_{n} x$, i.e.,

$$
\lim _{n \rightarrow \infty}\left\|(\gamma I-\mathbf{Z}) x-\sum_{i=1}^{n}\left(\gamma-\lambda_{i}\right)\left\langle x, e_{i}\right\rangle e_{i}\right\|=0 .
$$

Since $\left\{e_{i}\right\}$ is a complete orthonormal set, we have

$$
x=\sum_{i=1}^{\infty}\left\langle x, e_{i}\right\rangle e_{i} .
$$


Hence, taking the limit $n \rightarrow \infty$ in the triangle inequality

$$
\begin{aligned}
& \left\|(\gamma I-\mathbf{Z}) x-\sum_{i=1}^{n}\left(\gamma-\lambda_{i}\right)\left\langle x, e_{i}\right\rangle e_{i}\right\| \\
& \quad=\left\|\gamma\left(x-\sum_{i=1}^{n}\left\langle x, e_{i}\right\rangle e_{i}\right)-\left(\mathbf{Z} x-\sum_{i=1}^{n} \lambda_{i}\left\langle x, e_{i}\right\rangle e_{i}\right)\right\| \\
& \quad \leq \gamma\left\|x-\sum_{i=1}^{n}\left\langle x, e_{i}\right\rangle e_{i}\right\|+\left\|\mathbf{Z} x-\sum_{i=1}^{n} \lambda_{i}\left\langle x, e_{i}\right\rangle e_{i}\right\|
\end{aligned}
$$

we obtain (64) by (63) and (65).

Now, we return to the proof of Lemma 1. Since $Y \in \mathcal{N}$ and $\mathrm{X}^{*} \mathbf{Y X} \in \mathcal{N}$ by assumption, it follows from the above arguments that there exist complete orthonormal sets $\left\{e_{1}, e_{2}, \ldots\right\}$ and $\left\{f_{1}, f_{2}, \ldots\right\}$ consisting of the eigenvectors of $\mathbf{Y}$ and $\mathbf{X}^{*} \mathbf{Y X}$, respectively, such that for any $x \in \mathcal{M}, \mathbf{Y} x$ and $\mathrm{X}^{*} \mathbf{Y X} x$ can be expressed respectively as

$$
\begin{array}{r}
\mathbf{Y} x=\sum_{i=1}^{\infty} \mu_{i}\left\langle x, e_{i}\right\rangle e_{i} \\
\mathbf{X}^{*} \mathbf{Y X} x=\sum_{i=1}^{\infty} \nu_{i}\left\langle x, f_{i}\right\rangle f_{i}
\end{array}
$$

where $\mu_{i}$ is the eigenvalues of $\mathbf{Y}$ corresponding to $e_{i}$, and $\nu_{i}$ is the eigenvalue of $\mathbf{X}^{*} \mathbf{Y X}$ corresponding to $f_{i}$. Let $s$ and $t$ be the numbers of the negative eigenvalues of $\mathbf{Y}$ and $\mathbf{X}^{*} \mathbf{Y X}$, respectively. Here, we aim to show $s=t$. To this end, suppose that $s<t$, and assume without loss of generality that $\mu_{1}, \ldots, \mu_{s}<0$ while $\mu_{s+i} \geq 0(i \geq 1)$, and $\nu_{1}, \ldots, \nu_{t}<0$ while $\nu_{t+i} \geq 0(i \geq 1) .^{2}$ Also, consider the following linear equation for $\left[a_{1}, \ldots a_{t}\right]^{T}$ :

$$
\left[\begin{array}{ccc}
\left\langle\mathbf{X} f_{1}, e_{1}\right\rangle & \ldots & \left\langle\mathbf{X} f_{t}, e_{1}\right\rangle \\
\vdots & & \vdots \\
\left\langle\mathbf{X} f_{1}, e_{s}\right\rangle & \ldots & \left\langle\mathbf{X} f_{t}, e_{s}\right\rangle
\end{array}\right]\left[\begin{array}{c}
a_{1} \\
\vdots \\
a_{t}
\end{array}\right]=O .
$$

Then, as an under-determined equation, there exists a nontrivial solution $\left[a_{1}, \ldots, a_{t}\right]^{T}$ to (69). Let $z=\sum_{j=1}^{t} a_{j} f_{j}$. Since

$$
\left\langle z, f_{i}\right\rangle=\left\langle\sum_{j=1}^{t} a_{j} f_{j}, f_{i}\right\rangle= \begin{cases}a_{i} & 1 \leq i \leq t \\ 0 & t<i\end{cases}
$$

it follows from (68) that

$$
\mathbf{X}^{*} \mathbf{Y X} z=\sum_{i=1}^{\infty} \nu_{i}\left\langle z, f_{i}\right\rangle f_{i}=\sum_{i=1}^{t} \nu_{i} a_{i} f_{i} .
$$

Therefore, again from (70), we have

$$
\left\langle\mathbf{X}^{*} \mathbf{Y} \mathbf{X} z, z\right\rangle=\sum_{i=1}^{t} \nu_{i} a_{i}\left\langle f_{i}, z\right\rangle=\sum_{i=1}^{t} \nu_{i}\left|a_{i}\right|^{2}<0 .
$$

On the other hand, for $1 \leq i \leq s,\left\langle\mathbf{X} z, e_{i}\right\rangle=$ $\sum_{j=1}^{t} a_{j}\left\langle\mathbf{X} f_{j}, e_{i}\right\rangle=0$ by (69). This, together with (67), implies

$$
\mathbf{Y X} z=\sum_{i=1}^{\infty} \mu_{i}\left\langle\mathbf{X} z, e_{i}\right\rangle e_{i}=\sum_{i=s+1}^{\infty} \mu_{i}\left\langle\mathbf{X} z, e_{i}\right\rangle e_{i}
$$

${ }^{2}$ Note that the convergences of (67) and (68) are unconditional, i.e., these limits are invariant under reordering of the terms. See [22, Corollary 5.17.11].
Therefore, we have

$$
\begin{aligned}
\langle\mathbf{Y X} z, \mathbf{X} z\rangle & =\sum_{i=s+1}^{\infty} \mu_{i}\left\langle\mathbf{X} z, e_{i}\right\rangle\left\langle e_{i}, \mathbf{X} z\right\rangle \\
& =\sum_{i=s+1}^{\infty} \mu_{i}\left|\left\langle\mathbf{X} z, e_{i}\right\rangle\right|^{2} \geq 0
\end{aligned}
$$

This, together with (71), contradicts $\left\langle\mathbf{X}^{*} \mathbf{Y X} z, z\right\rangle=$ $\langle\mathbf{Y X} z, \mathbf{X} z\rangle$. Therefore, $s \geq t$. We can establish $s \leq t$ in a similar manner, and, hence, we obtain $s=t$.

Finally, we have to show that the multiplicity of the zero eigenvalue is invariant under infinite-dimensional congruent transformations. However, this is immediate since we can readily see that the dimension of the eigenspace corresponding to the zero eigenvalue is invariant. This completes the proof.

Proof of Lemma 2: Suppose that $\mathbf{Y}=\left[\begin{array}{ll}\mathbf{Q} & \mathbf{S} \\ \mathbf{S}^{*} & \mathbf{R}\end{array}\right]=\gamma I-$ $\left[\begin{array}{cc}\underline{\underline{\mathbf{Q}}} & \underline{\mathbf{S}} \\ \underline{\underline{\mathbf{R}}} & \underline{\mathbf{R}}\end{array}\right]$ and $\mathbf{X}=\left[\begin{array}{ll}{ }_{-\mathbf{R}^{-1}} \mathbf{S}^{*} & O \\ I\end{array}\right]$ where $\left[\begin{array}{ll}\underline{\underline{\mathbf{Q}}} & \underline{\underline{\mathbf{S}}} \\ \underline{\underline{\mathbf{R}}}\end{array}\right] \in \mathcal{W}$. Then

$\mathbf{X}^{*} \mathbf{Y X}=\left[\begin{array}{cc}\mathbf{Q}-\mathbf{S R}^{-1} \mathbf{S}^{*} & O \\ O & \mathbf{R}\end{array}\right]=\gamma I-\left[\begin{array}{cc}\underline{\mathbf{Q}}+\underline{\mathbf{S}}^{-1} \underline{\mathbf{S}}^{*} & O \\ O & \underline{\mathbf{R}}\end{array}\right]$.

Similarly, by letting $\mathbf{X}=\left[\begin{array}{cc}I & -\mathbf{Q}^{-1} \mathbf{S} \\ O & I\end{array}\right]$, we obtain

$$
\mathbf{X}^{*} \mathbf{Y X}=\left[\begin{array}{cc}
\mathbf{Q} & O \\
O & \mathbf{R}-\mathbf{S}^{*} \mathbf{Q}^{-1} \mathbf{S}
\end{array}\right]=\gamma I-\left[\begin{array}{cc}
\underline{\mathbf{Q}} & O \\
O & \underline{\mathbf{R}}+\underline{\mathbf{S}}^{*} \mathbf{Q}^{-1} \underline{\mathbf{S}}
\end{array}\right]
$$

Therefore, from Lemma 1, the proof becomes complete if we show that $\underline{\mathbf{Q}} \in \mathcal{W}, \underline{\mathbf{R}} \in \mathcal{W}, \underline{\mathbf{S}}^{-1} \underline{\mathbf{S}}^{*} \in \mathcal{W}$, and $\underline{\mathbf{S}}^{*} \mathbf{Q}^{-1} \underline{\mathbf{S}} \in$ $\mathcal{W}$. However, this is immediate since $\left[\begin{array}{ll}\underline{\mathbf{Q}} & \underline{\mathbf{S}}\end{array}\right] \in \mathcal{W}$ if and only if $\underline{\mathbf{Q}} \in \mathcal{W}, \underline{\mathbf{R}} \in \mathcal{W}$, and $\underline{\mathbf{S}}$ is compact.

Q.E.D.

Proof of Lemma 3: By definition, $\mathbf{Y}>O$ if and only if $\langle\mathbf{Y} x, x\rangle>0$ for every nonzero $x \in \mathcal{M}$. However, since $\langle\mathbf{Y} x, x\rangle=\sum_{i=1}^{\infty} \mu_{i}\left|\left\langle x, e_{i}\right\rangle\right|^{2}$ by (67), we can see that $\mathbf{Y}>O$ if and only if $\mu_{i}>0(\forall i)$. This completes the proof. Q.E.D.

Proof of Lemma 4: $X=\left[\begin{array}{cc}O & A \\ A^{*} & O\end{array}\right]$ is similar to $-X$ since $-X=J^{-1} X J$ where $J=\left[\begin{array}{cc}I & O \\ O & -I\end{array}\right]$. Therefore, the set of the eigenvalues of $X$ and that of $-X$ coincide, including multiplicities. By this, together with the assumption that $A$ is invertible, we obtain $\mathrm{N}(X)=(0, n)$.

Q.E.D.

\section{ACKNOWLEDGMENT}

The authors thank Y. Yamamoto for his valuable comments and suggestions. They also thank S. Kimura and T. Mifune for their assistance in the numerical computations of the example.

\section{REFERENCES}

[1] M. Araki, Y. Ito, and T. Hagiwara, "Frequency response of sampled-data systems," Automatica, vol. 32, no. 4, pp. 483-497, 1996.

[2] B. D. O. Anderson and J. B. Moore, Optimal Control-Linear Quadratic Method. Englewood Cliffs, NJ: Prentice-Hall, 1990.

[3] S. Boyd, V. Balakrishnan, and P. Kabamba, "A bisection method for computing the $H_{\infty}$ norm of a transfer matrix and related problems," Math. Control, Signals, Syst., vol. 2, pp. 207-220, 1989.

[4] B. A. Bamieh and J. B. Pearson, "The $\mathrm{H}_{2}$ problem for sampled-data systems," Syst. Control Lett., vol. 19, no. 1, pp. 1-12, 1992.

[5] _ "A general framework for linear periodic systems with applications to $H_{\infty}$ sampled-data control," IEEE Trans. Automat. Contr., vol. 37, pp. 418-435, Apr. 1992. 
[6] T. Chen and B. A. Francis, Optimal Sampled-Data Control Systems. New York: Springer-Verlag, 1996.

[7] G. Dullerud, "Computing the $L_{2}$-induced norm of a compression operator," Syst. Control Lett., vol. 37, no. 2, pp. 87-91, 1999.

[8] G. Dullerud and K. Glover, "Robust stabilization of sampled-data systems to structured LTI perturbations," IEEE Trans. Automat. Contr., vol. 38, pp. 1497-1508, Oct. 1993.

[9] J. S. Freudenberg, R. H. Middleton, and J. H. Braslavsky, "Inherent design limitations for linear sampled-data feedback systems," Int. J. Control, vol. 61, no. 6, pp. 1387-1421, 1995.

[10] T. Hagiwara, "Nyquist stability criterion and positive realness of sampled-data systems," in Proc. 2000 Amer. Control Conf., pp. 958-962.

[11] T. Hagiwara and M. Araki, "FR-operator approach to the $H_{2}$ analysis and synthesis of sampled-data systems," IEEE Trans. Automat. Contr., vol. 40, pp. 1411-1421, Aug. 1995.

[12] _ _ "Robust stability of sampled-data systems under possibly unstable additive/multiplicative perturbations," IEEE Trans. Automat. Contr., vol. 43, pp. 1340-1346, Sept. 1998.

[13] T. Hagiwara, Y. Ito, and M. Araki, "Computation of the frequency response gains and $H_{\infty}$-norm of a sampled-data system," Syst. Control Lett., vol. 25 , no. 4, pp. 281-288, 1995.

[14] T. Hagiwara, M. Suyama, and M. Araki, "Upper and lower bounds of the frequency response gain of sampled-data systems," in Proc. 37th Conf. Decision Control, 1998, pp. 319-324.

[15] S. Hara, H. Fujioka, P. P. Khargonekar, and Y. Yamamoto, "Computational aspects of gain-frequency response for sampled-data systems," in Proc. 34th Conf. Decision Control, 1995, pp. 1784-1789.

[16] Y. Hayakawa, S. Hara, and Y. Yamamoto, " $H_{\infty}$ type problem for sampled-data control systems-A solution via minimum energy characterization," IEEE Trans. Automat. Contr., vol. 39, pp. 2278-2284, Nov. 1994.

[17] Y. Ito, T. Hagiwara, and M. Araki, " $H_{\infty}$ problem of sampled-data systems viewed from FR-operators," in Proc. 22nd SICE Symp. Control Theory, 1993, pp. 7-12.

[18] P. T. Kabamba and S. Hara, "Worst-case analysis and design of sampled-data control systems," IEEE Trans. Automat. Contr., vol. 38, pp. 1337-1357, Sept. 1993.

[19] P. P. Khargonekar and N. Sivashankar, " $H_{2}$ optimal control for sampled-data systems," Syst. Control Lett., vol. 17, pp. 425-436, 1991.

[20] B. P. Lampe and Y. N. Rosenwasser, " $H_{2}$-optimization of sampled-data systems with neutral continuous-time plants," in Proc. 37th Conf. Decision Control, 1998, pp. 853-854.

[21] A. Megretski and A. Rantzer, "System analysis via integral quadratic constraints," IEEE Trans. Automat. Contr., vol. 42, pp. 819-830, June 1997.

[22] A. W. Naylor and G. R. Sell, Linear Operator Theory in Engineering and Science. New York: Springer-Verlag, 1982.

[23] Y. Oishi, "A bound of conservativeness in sampled-data robust stabilization and its dependence on sampling periods," Syst. Control Lett., vol. 32, no. 1, pp. 11-19, 1997

[24] A. Rantzer, "On the Kalman-Yakubovich-Popov lemma," Syst. Control Lett., vol. 28, no. 1, pp. 7-10, 1996.

[25] Y. N. Rosenvasser, K. Y. Polyakov, and B. P. Lampe, "Application of Laplace transformation for digital redesign of continuous control systems," IEEE Trans. Automat. Contr., vol. 44, pp. 883-886, Apr. 1999.

[26] K. Sugimoto and M. Suzuki, "On $\gamma$-positive real sampled-data control systems," in Proc. 13th Int. Symp. Mathematical Theory Networks Systems, Padova, Italy, 1998, pp. 409-412.

[27] _ - "On $\gamma$-positive real sampled-data control systems and their phase property" (in Japanese), Trans. Soc. Instrument Control Engineers, vol. 35, no. 1, pp. 71-76, 1999.

[28] H. T. Toivonen, "Sampled-data control of continuous-time systems with an $H_{\infty}$ optimality criterion," Automatica, vol. 28 , no. 1 , pp. $45-54$, 1992.

[29] Y. Yamamoto, "On the state space and frequency domain characterization of $H_{\infty}$ norm of sampled-data systems," Syst. Control Lett., vol. 21, no. 2, pp. 163-172, 1993.

[30] Y. Yamamoto and M. Araki, "Frequency responses for sampled-data systems-Their equivalence and relationships," Linear Alg. Appl., vol. 205-206, pp. 1319-1339, 1994.

[31] Y. Yamamoto, H. Fujioka, and P. P. Khargonekar, "Signal reconstruction via sampled-data control with multirate filter banks," in Proc. 36th Conf. Decision Control, 1997, pp. 3395-3400.
[32] Y. Yamamoto and P. P. Khargonekar, "Frequency response of sampled-data systems," IEEE Trans. Automat. Contr., vol. 41, pp. 166-176, Feb. 1996.

[33] Y. Yamamoto, A. G. Madievski, and B. D. O. Anderson, "Computation and convergence of frequency response via fast sampling for sampled-data control systems," in Proc. 36th Conf. Decision Control, 1997, pp. 2157-2162.

[34] N. Young, An Introduction to Hilbert Space. Cambridge, U.K.: Cambridge University Press, 1988.

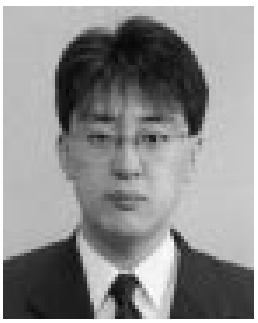

Yoshimichi Ito was born in Gifu, Japan, in 1967. He received the B.E. and M.E. degrees in electrical engineering from Kyoto University, Kyoto, Japan, in 1990 and 1992, respectively.

Since 1994, he has been with the Department of Communications Engineering, Osaka University, Osaka, Japan. His research interests include sampled-data control and digital signal processing.

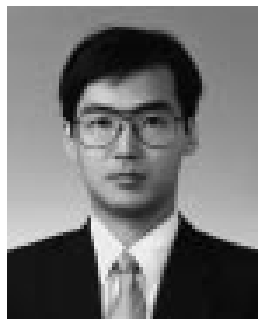

Tomomichi Hagiwara (M'96) was born in Osaka, Japan, in 1962. He received the B.E., M.E., and Ph.D. degrees in electrical engineering from Kyoto University, Kyoto, Japan, in 1984, 1986 and 1990, respectively.

Since 1986, he has been with the Department of Electrical Engineering, Kyoto University, where he is currently an Associate Professor. His research interests include sampled-data control, nonlinear/robust stability, two-degrees-of-freedom control systems, and dynamical system theory.

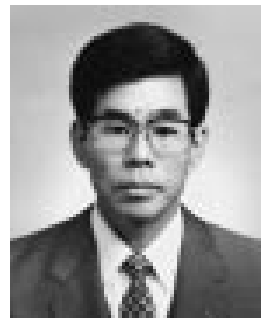

Hajime Maeda (S'66-M'71) was born in Osaka, Japan, in 1943. He received the B.E., M.E. and Ph.D. degrees all in communications engineering from Osaka University, Osaka, Japan, in 1966, 1968, and 1971, respectively.

In 1971, he joined the Department of Mechanical Engineering at the Faculty of Engineering Science, Osaka University. From 1983 to 1984, he was a Visiting Associate Professor at the University of Waterloo, ON, Canada. Since 1993, he has been with the Department of Communications Engineering, Osaka University, where he is currently a Professor. His main research interest is in the area of control theory, nonlinear analysis, and signal processing.

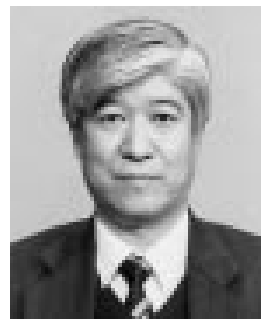

Mituhiko Araki (M'80) was born in 1943. He received the B.E., M.E., and Ph.D. degrees, all in electronic engineering, from Kyoto University, Kyoto, Japan, in 1966, 1968, and 1971, respectively.

Since 1971, he has been with the Department of Electrical Engineering, Kyoto University, where he is currently a Professor. He has been engaged in researches on control theory and its applications, with topics such as stability of composite systems, control of plants with pure delays, control of synchronous generators, two-degree-of-freedom PID controllers, two-degree-of-freedom LQI controllers, multirate digital control, frequency-domain theory of sampled-data systems, scheduling problems, blood pressure control of patients under surgical operation, blood sugar control, etc. He is a Council Member of IFAC and an Editor of Automatica. 